\title{
Turbulence Measurements of Rectangular Nozzles with Bevel
}

\author{
James Bridges* and Mark P. Wernet ${ }^{\dagger}$ \\ NASA Glenn Research Center \\ Cleveland, $\mathrm{OH}$
}

\begin{abstract}
This paper covers particle image velocimetry measurements of a family of rectangular nozzles with aspect ratios 2,4 , and 8 , in the high subsonic flow regime. Far-field acoustic results, presented previously, showed that increasing aspect ratios increased the high frequency noise, especially directed in the polar plane containing the minor axis of the nozzle. The measurements presented here have important implications in the modeling of turbulent sources for acoustic analogy theories. While the nonaxisymmetric mean flow from the rectangular nozzles can be studied reliably using computational solutions, the nonaxisymmetry of the turbulent fluctuations, particularly at the level of velocity components, cannot; only measurements such as these can determine the impact of nozzle geometry on acoustic source anisotropy. Additional nozzles were constructed that extended the wide lip on one side of these nozzles to form beveled nozzles. The paper first documents the velocity fields, mean and variance, from the round, rectangular, and beveled rectangular nozzles at high subsonic speeds. A second section introduces measures of the isotropy of the turbulence, such as component ratios and lengthscales, first by showing them for a round jet and then for the rectangular nozzles. From these measures the source models of acoustic analogy codes can be judged or modified to account for these anisotropies.
\end{abstract}

\footnotetext{
Nomenclature

$D_{j} \quad=$ diameter, equivalent area

$h \quad=$ short dimension of rectangular nozzle exit
}

\footnotetext{
Acoustics Branch, MS 54-3; AIAA Associate Fellow

† Instrumentation Branch, MS 77-1; AIAA Senior Fellow
} 


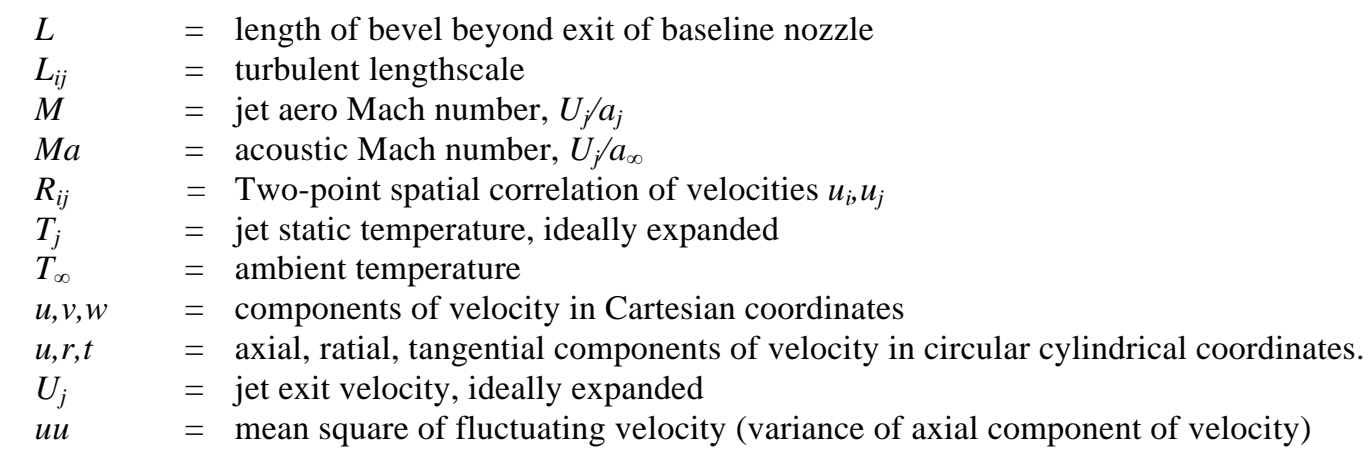

\section{Introduction}

The Supersonics Project of NASA’s Fundamental Aeronautics Program is developing technologies to enable civilian supersonics aircraft. Two of the top challenges to such vehicles have been sonic boom and noise around airports. One approach for reducing sonic boom is to avoid abrupt changes in aircraft cross-sectional area, hence embedding the propulsion. For the propulsion system to vary its bypass ratio to meet both cruise performance and airport noise requirements requires variable area nozzles. Both of these aspects of supersonic aircraft design point to the need for non-axisymmetric inlets and nozzles. Many existing noise prediction tools explicitly assume axisymmetric nozzle geometry and may not predict noise accurately from such inlets and nozzles; others simply have not been demonstrated. As part of its exploration of nozzle concepts for quiet civilian supersonic aircraft, the NASA Supersonics Project created a family of high aspect ratio nozzles, the Extensible Rectangular Nozzles (ERN).

The initial goal of testing of the ERN was to determine whether high aspect ratio nozzles showed promise for noise reduction, to gather basic noise data for creating empirical models, and to provide flow data for advanced noise prediction code development. The promise of acoustic analogy codes is that the impact of geometric variations in nozzle shape can be captured by Reynolds-Averaged Navier-Stokes (RANS) solutions with enough fidelity to predict their acoustic impact. Most acoustic analogy formulations ${ }^{1,2}$ require that the detailed source terms, often involving space-time correlations of either velocity or Reynolds stresses, can be modeled given only the overall turbulent kinetic energy and a dissipation variable. Acoustic source amplitude, time and length scales are derived from these flow parameters to construct the model. However, remaining undefined is the split of the kinetic energy into the spatial components of the correlation matrix. Either explicitly or implicitly, the relationships between cross-stream and axial components of velocity are also modeled, often based on rather simple ideas of the isotropy 
of turbulence, a la Batchelor ${ }^{3}$. Given the significant number of modeling coefficients involved, such models may work as long as the jet flow does not deviate far from that for which it was calibrated (usually a round jet). But when the geometry is strongly nonaxisymmetric will the turbulence continue to have the same split in its directional components? And will this break the internal models for the source terms? Or are all jet flows sufficiently the same in the isotropy of the turbulence that a common model will suffice?

The particle image velocimetry (PIV) data reported here serves to validate the computational fluid dynamics solutions being used in the development of acoustic prediction codes. By choosing nozzle geometries with relatively simple shape, robust CFD can be done easily. Secondly, it serves as a test case for checking whether the isotropy of the turbulence is significantly affected by the overall nozzle geometry. Rectangular nozzles represent relatively strong non-axisymmetry at a very low azimuthal order, with significant portions of the flow having different amounts of azimuthal curvature - very different from round jets. They are also of interest in applications, making this study useful on several levels.

Design of the Extensible Rectangular Nozzle (ERN) model system using computational fluid dynamics was documented in Reference [4]. The main design criterion was to create a family of rectangular nozzles that had uniform velocity at their exit plane. The nozzles were tested for farfield noise in December 2010 and acoustic results reported in Reference [5]. Detailed velocity field measurements are presented in this paper.

Other researchers have investigated rectangular nozzles previously. One example is the work of General Electric Co., who acquired data on a 6:1 'slot' nozzle at their Corporate Research and Development labs in the late 1970's ' . Georgia Tech Research Institute (GTRI) made a more thorough investigation $^{7}$ of rectangular jets, giving results for aspect ratios 1.5, 4, and 8. Little flow field information was available in these tests.

\section{Facility, Model Hardware, Instrumentation, and Flow Conditions}

\section{A. Facility}

The test was conducted on the Small Hot Jet Acoustic Rig (SHJAR, pronounced with a silent ' $J$ '). The rig is located in the Aeroacoustic Propulsion Laboratory (AAPL) at the NASA Glenn Research Center in Cleveland, Ohio. The SHJAR was developed to test jet noise reduction concepts at a low technology readiness level (TRL 1-3) and at minimum expense, and to conduct 
fundamental studies of jet noise and jet turbulent flow fields. Since 2003 more than ten PIV test campaigns have been conducted in the SHJAR. Of significance to this report is the compilation of particle image velocimetry (PIV) results found in Reference 8 and the synopsis of PIV methodology described in Reference 9. Details specific to the test being reported are given below.

\section{B. Model Hardware}

The nozzles being tested were designed with a few criteria in mind. The key criteria were that the flow at the exit plane of the nozzle be uniform without swirl from the round-to-rectangular transition; that there be no separations on internal surfaces of the nozzles, that the nozzle lip be very thin to avoid lip-separation noise, and that the nozzles for a given aspect ratio have the same internal shape independent of the external features. The details of the design process, including CFD and mechanical stress analysis, are covered in a conference paper [4].

The nozzles that were eventually fabricated for this test are shown in Table 1. Three aspect ratios were chosen, 2:1, 4:1, and 8:1 with height $h$ and width $w$ giving an equivalent diameter of 2.14 inches $(545 \mathrm{~mm})$. Beyond the basic rectangular nozzles, beveled designs with two different extension lengths $L$ were constructed for each aspect ratio. The lengths of the lower edge of beveled nozzle were made in increments relative to the minimum dimension of the rectangular nozzles, as given in Table 1. Other variations in nozzle design were constructed and tested, including chevrons on the wide sides of the rectangular nozzle, but these results will not be presented here for brevity. Finally, PIV data was acquired with a round nozzle of similar size (2 inch diameter) that has been tested extensively before ( $c f$. Reference 8; SMC000). Beyond being a reference for these rectangular nozzles, the SMC000 data allowed a check that the rig and instrumentation were performing as before. 
Table 1 Design features of nozzle hardware.

Basic nozzle, 2:1, NA2Z 1.34"x2.68"

Basic nozzle, $4: 1$, 0.948 "x3.79"

NA4Z

\section{( \\ Basic nozzle, 8:1, $0.671 " x 5.36 "$} NA8Z

\section{Bevel nozzle, $2: 1,1.3$ " ext $(L / h=1)$ $\left(L / D_{j}=0.625\right)$} NA2B1

Bevel nozzle, 2:1, 2.7" ext $(L / h=2)$ $\left(L / D_{j}=1.25\right)$

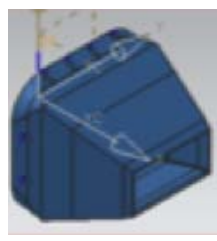

Bevel nozzle, $4: 1,1.3 "$ ext $(L / h=1.4)$ $\left(L / D_{j}=0.625\right)$

NA4B1
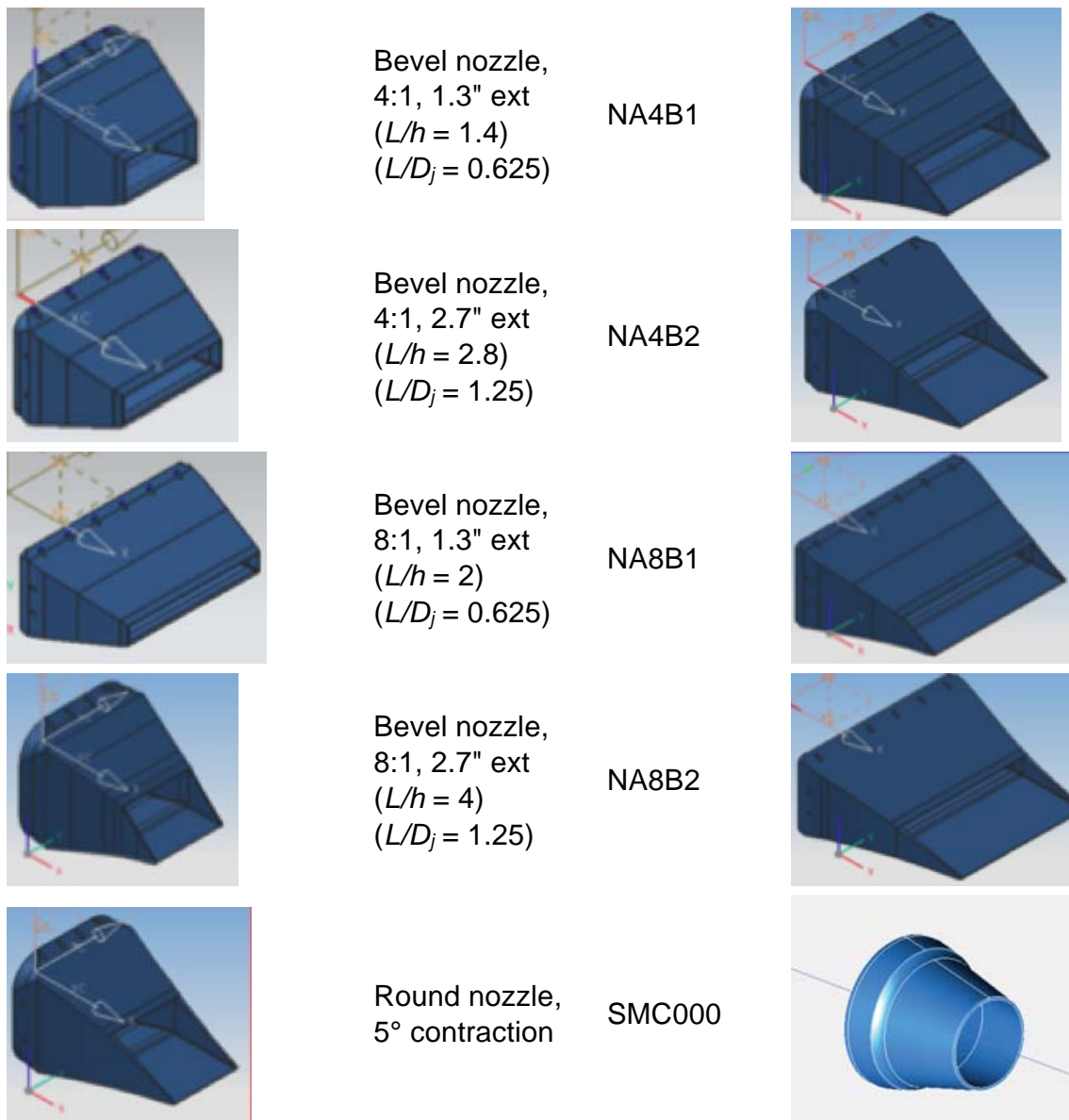

Bevel nozzle, $4: 1,2.7 "$ ext $(L / h=2.8)$ $\left(L / D_{j}=1.25\right)$

Bevel nozzle, 8:1, 1.3" ext $(L / h=2)$ $\left(L / D_{j}=0.625\right)$

Bevel nozzle, 8:1, 2.7" ext $(L / h=4)$ $\left(L / D_{j}=1.25\right)$

NA8B2

NA4B2

NA8B1
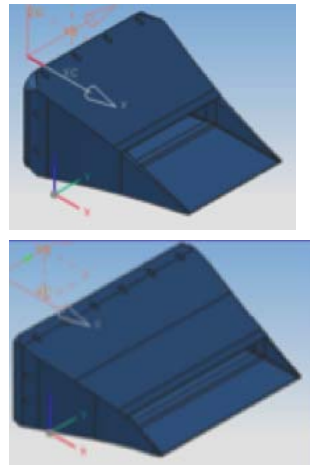

Round nozzle, $5^{\circ}$ contraction

SMC000

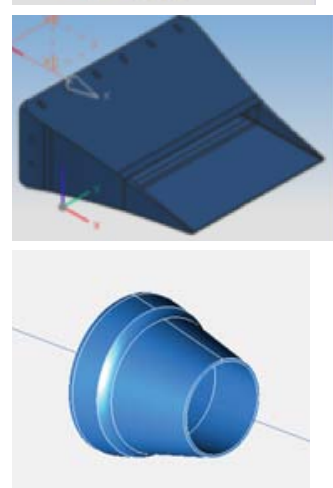

Because the test program had such a large number of geometries, and a large number of orientations for each of these geometries, only a cursory number of flow setpoints were explored. The flow setpoints were extracted from a much larger set that spans the range of velocity and temperatures previously tested on SHJAR for round and chevron nozzles. This subset was focused on the high subsonic flows that are likely to be important in civilian supersonic aircraft. In this paper data will be presented for an unheated flow at acoustic Mach number 0.9. All cases were also acquired for at heated flow at static temperature ratio of 2.3 , but these data did not yield any different conclusions.

\section{Instrumentation}

PIV data were acquired in two modes: two-component streamwise, and three-component cross-stream. Both used the same lasers, cameras, and image acquisition equipment. Seeding of the jet flow was done using a $\mathrm{pH}$-stabilized dispersion of $0.4 \mu \mathrm{m}$ alumina particles in alcohol, which was atomized into the flow upstream of the SHJAR settling chamber. Seeding of the 
ambient air was provided by a pair of $\mathrm{MDG}^{\mathrm{TM}}$ oil foggers, located in the framework of the SHJAR and directed around the jet plume by a pair of $1 \mathrm{~m}$-diameter room fans.

\section{3-D Stereo PIV Configuration}

The Stereo Particle Image Velocimetry (PIV) system was configured to provide cross-stream measurements of the three-component velocity field from the test article. The entire PIV system was mounted on a large traverse system to facilitate performing plane surveys of the flow field. The PIV system employed two high-resolution (4008 x 2672 pixels) cameras equipped with 7inch focal length lenses and 0.31-inch extension tubes to provide a 11.8 x 14.2 inch field of view. The cameras were mounted downstream of the model exit plane at nominally $\pm 45^{\circ}$ from the nozzle centerline. Stereo PIV calibrations were performed using a single plane target translated to 9 axial positions over a \pm 0.1 inch range. A $4^{\text {th }}$-order polynomial was used in the calibration and a calibration verification operation was employed to insure the calibration overlapped the laser light sheet plane. The measurement plane was illuminated using a dual head $400 \mathrm{~mJ} / \mathrm{pulse}$ $\mathrm{Nd}$ :YAG laser system. The laser beams were formed into 0.05 x 14 inch light sheets using cylindrical and spherical lenses. Both cameras were connected to a single computer system via a CameraLink $^{\mathrm{TM}}$ PCI card and the 400 frame pair data sequences were acquired and streamed to disk at a rate of 2 frame-pairs/camera/sec.

Since the AAPL is open to the environment during testing, the facility could not be operated in complete darkness. To accommodate this situation, optical backdrops for the cameras were provided. The backdrops were offset a suitable distance to minimize any influence on the ambient seeded flow distribution. The framestraddle cameras used in the PIV systems incorporate fast-acting electromechanical shutters in front of the CCD arrays to minimize the duration of the frame-straddled second exposures to nominally 12 milliseconds (down from the camera fixed 240 millisecond $2^{\text {nd }}$ frame integration period). The complete stereo PIV system, including all cameras and backdrops, data acquisition computers, laser hardware and optics were rigidly mounted on a large axial traverse located downstream of the nozzle exit plane, which maintained a fixed position during each nozzle test. The travel range of the traverse was approximately 98 inches, with a positioning accuracy of 0.04 inches. Re-zeroing of the traverse to coincide with the trailing edge of individual nozzles was aided by the installation of a calibration target, required in stereo PIV, on a fixture secured to the traverse. When operating at elevated temperature setpoints, with an accompanying axial growth of the entire test rig 
following cold start-up, traverse re-zeroing adjustment was required and performed immediately after reaching steady state flow conditions by optical means, using the current camera nozzle views to known reference locations at the nozzle exit planes.

\section{2-D Streamwise PIV Configuration}

To maximize the field of view while maintaining high spatial resolution PIV vector maps, a dual side-by-side camera configuration was used. The 4008x2672 pixel stereo PIV cameras were used with the 4008-pixel axis oriented vertically with fields of view that overlapped by 1 inch. A PIV calibration target was used to calibrate and register the two cameras. The physical registration of the two cameras was used in the setup of the vector processing grids in the left and right camera images so that no interpolation was required in the merging of the left/right vector maps. The final merged camera vector map covered an area of 15 x 12.8 inches.

\section{Vector Processing}

Velocity vector maps for each camera were computed from the image pairs using the in-house PIVPROC software. The software utilized conventional multi-pass PIV cross-correlation processing algorithms and incorporated error detection based on image correlation signal to noise ratio. First pass interrogation region sizes of $64 \times 64$ pixels on 32 pixel centers and final pass interrogation region sizes of 32 x 32 pixels on 16 pixel centers were used to process image pairs from the cameras in both stereo configurations. For the cross-flow measurement planes near the nozzle, Symmetric Phase Only Filtering (SPOF) was employed to reduce the effects of flare light on the nozzle models directly behind the measurement planes. Without the SPOF processing, images with nozzles illuminated by laser flare light behind the plane of interest generally produced regions in and around the potential core flow with invalid vector measurements. The SPOF processing technique was not utilized with the 2-D streamwise measurements, as no images with nozzle models in the background were present. All of the data were processed using LogLut intensity filters and Subregion Distortion processing. For the 3-D PIV data, the left/right vector maps were processed with an additional in-house code to generate the 3-D vector maps.

\section{Geometries of measurement planes}

The origin of the coordinate system was the center of the exit plane of the basic rectangular nozzle. The streamwise PIV field of view was centered on the jet axis. Streamwise PIV data were acquired in two orthogonal planes for each basic rectangular nozzle corresponding to the two symmetry planes of the nozzle. Only the plane containing the minor axis (symmetry plane) 
of the beveled nozzles was measured. The beveled nozzles were mounted such that the longer edge was away from the source of the light sheet, allowing the sidewalls of the nozzle to shield the cameras from the reflection of the light sheet on the inner nozzle surface. The PIV system was traversed axially in increments of 11 ", creating a 1.5 " overlap between acquisitions, providing a total scanned field of 45 " with 4 acquisitions.

The cross-stream PIV had a nominal field of view of 12" x 14" per acquisition point. Data were acquired at 11 axial stations given in Table 2. This full matrix of locations applied to the baseline round and rectangular nozzles. Beveled nozzles extended downstream of the baseline exit plane and locations were dropped if they intersected the model. For instance, for the long bevel nozzles, the first three axial stations were not acquired. The first position available for each model was as close to the nozzle exit as possible.

Table 2 Axial locations of cross-stream PIV measurement planes.

\begin{tabular}{|c|c|c|c|c|}
\hline \hline index & $x / D_{i}$ & & index & $x / D_{i}$ \\
\hline \hline 1 & 0.10 & & 7 & 5.00 \\
2 & 0.70 & & 8 & 6.50 \\
3 & 1.10 & & 9 & 8.00 \\
4 & 1.40 & & 10 & 10.00 \\
5 & 2.00 & & 11 & 15.00 \\
6 & 3.50 & & & \\
\hline
\end{tabular}

\section{Results}

\section{A. Validation of design of contraction on rectangular nozzles}

Velocity data were acquired on a plane approximately 0.2 inches downstream of the exit of the rectangular nozzles to document the initial velocity profile of the jet. This is critical since different methods of transitioning from the round plenum to the rectangular exit generally produce swirls (typically found in the corners) and nonuniformity of the mean velocity.

In Figure 1 the exit plane is mapped for the three different aspect ratio baseline nozzles. The figure contains contour plots of mean axial velocity and mean axial vorticity (as limited by the spatial resolution of the velocity measurement) superimposed on the nozzle. The measurements of axial velocity have spatial resolution issues as the boundary layers are much smaller than the image fragment over which particle images are correlated to make a velocity measurement. The 2:1 nozzle shows some evidence of acceleration on the contracting walls, having a slightly higher velocity (2\%) near the wall than on the centerline. The 4:1 nozzle had a very uniform 
profile, but the 8:1 nozzle shows some evidence of having a velocity deficit across roughly $1 / 3$ of the long span.

Designing a nozzle with uniform mean exit velocity is not as difficult as not having axial vorticity. In the design of these nozzles RANS CFD often showed counter-rotating axial vortices in the corners due to the differential contractions of the horizontal and vertical walls. Several redesigns were required to remove these artifacts. In the data shown here the vorticity measured is often not concentrated in the corners, but along the small edge. And when the vorticity is concentrated at a nozzle corner it is asymmetrically paired as is typical of axial vortices produced by uneven acceleration of nozzle walls. For reference, the maximum $\omega_{1}$ vorticity levels in the cross-stream plots are 1/40th that of the cross-stream $\omega_{1}$ vorticity in the initial shear layer. The main objective of the design CFD effort was to achieve a uniform flow profile at the nozzle exit plane with no swirl. To the degree this could be measured, this objective was achieved. 


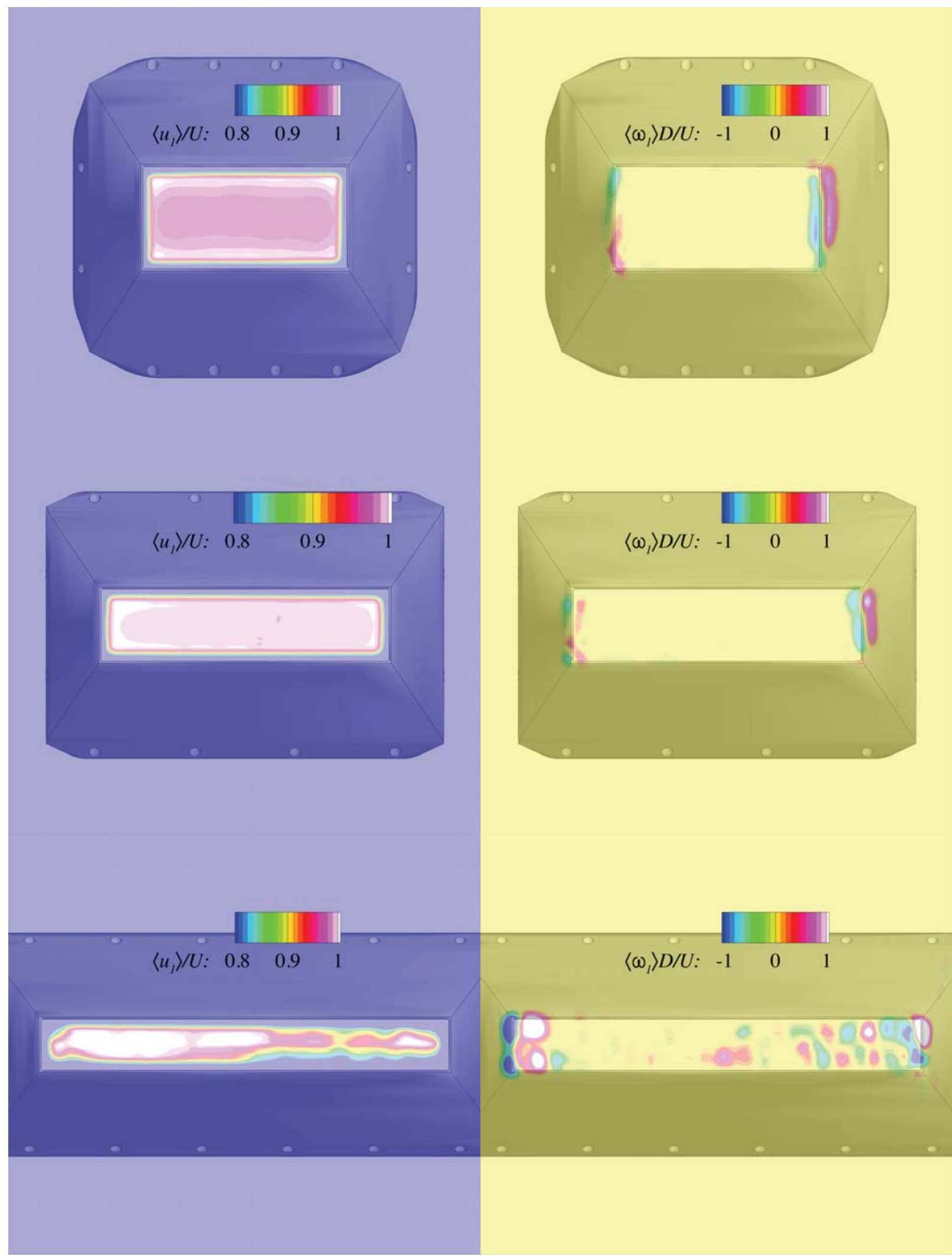

Figure 1 Mean axial velocity and axial vorticity as measured at the exit planes of the rectangular nozzles: 2:1 (top), $4: 1$ (middle), 8:1 (bottom). Unheated, $M a=0.9$ (setpoint 7) jet condition. 


\section{A. Mean and variance of velocity}

To get an overview of the flow fields which result from the different nozzles a series of contour plots have been prepared showing the mean and variance of axial velocity component in the measurement planes. In the following, mean and variance of velocities are defined as follows:

$$
\begin{aligned}
& U_{i}=\frac{1}{T} \int_{0}^{T} u_{i} d t \\
& u_{i} u_{i}=\frac{1}{T} \int_{0}^{T}\left(u_{i}-U_{i}\right)\left(u_{i}-U_{i}\right) d t ; \quad u_{i}^{\prime}=\sqrt{u_{i} u_{i}} .
\end{aligned}
$$

\section{Round nozzle}

For reference, and to provide a verification dataset for the PIV instrumentation used in this test, data from the round SMC000 nozzle is presented first. For the round nozzle only one streamwise plane was measured. This plane, and the series of cross-stream planes also measured for the unheated, $M a=0.9$ flow condition (setpoint 7) are given in Figure 2. The results are comparable to measurements made in previous years on this rig $^{8}$.
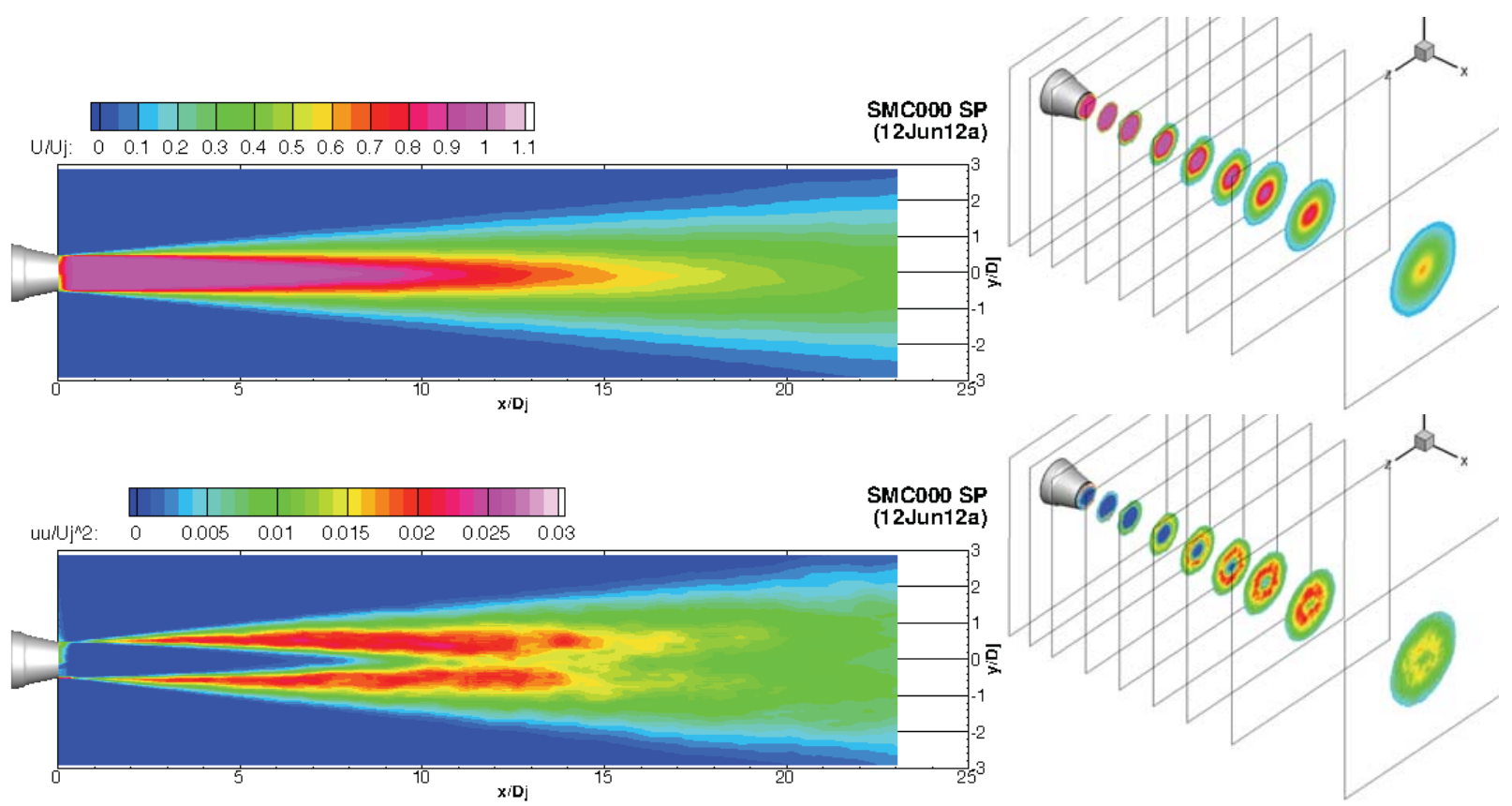

Figure 2 Mean (top) and variance (bottom) of axial velocity from round jet, Ma=0.9, unheated.

\section{Rectangular nozzles}

For the basic rectangular nozzles two streamwise planes were measured, one containing the minor axis $(x, y)$ and one containing the major axis $(x, z)$. In the Figures below, both mean and variance of axial velocity are shown in panels 
that include the two streamwise measurement planes $(y / D=0, z / D=0)$ and an isometric view with cross-stream planes at $\mathrm{x} / \mathrm{D}=0.1,0.7,1.1,1.4,2.0,3.5,5.0,6.5,8.0,10$, and 15 .

Figure 3 presents these data for the $2: 1$ nozzle, while

Figure 4 and Figure 5 present them for the 4:1 and 8:1 nozzles respectively.

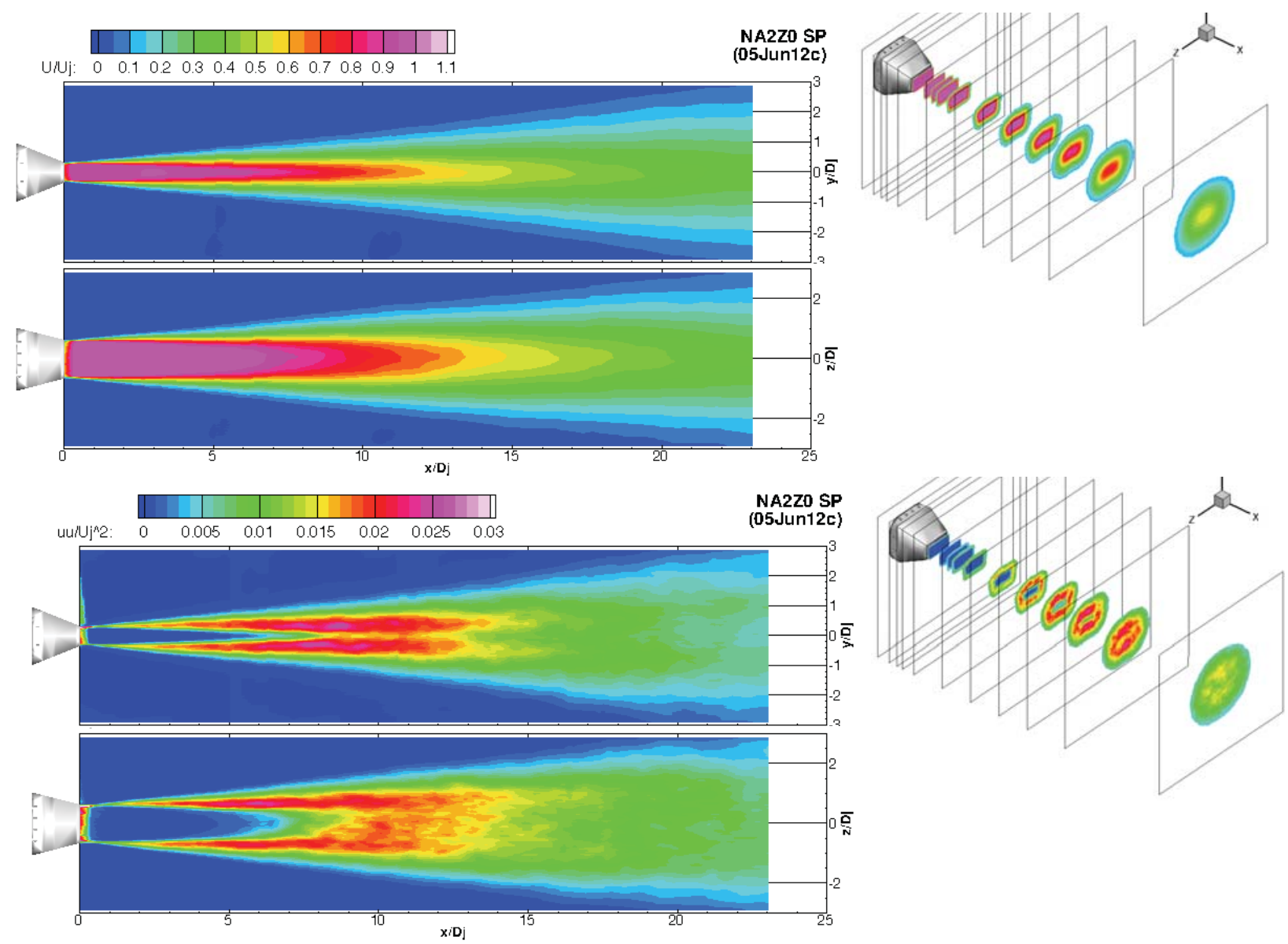

Figure 3 Mean (top) and variance (bottom) of axial velocity from baseline aspect ratio 2 rectangular jet, Ma=0.9, unheated. 


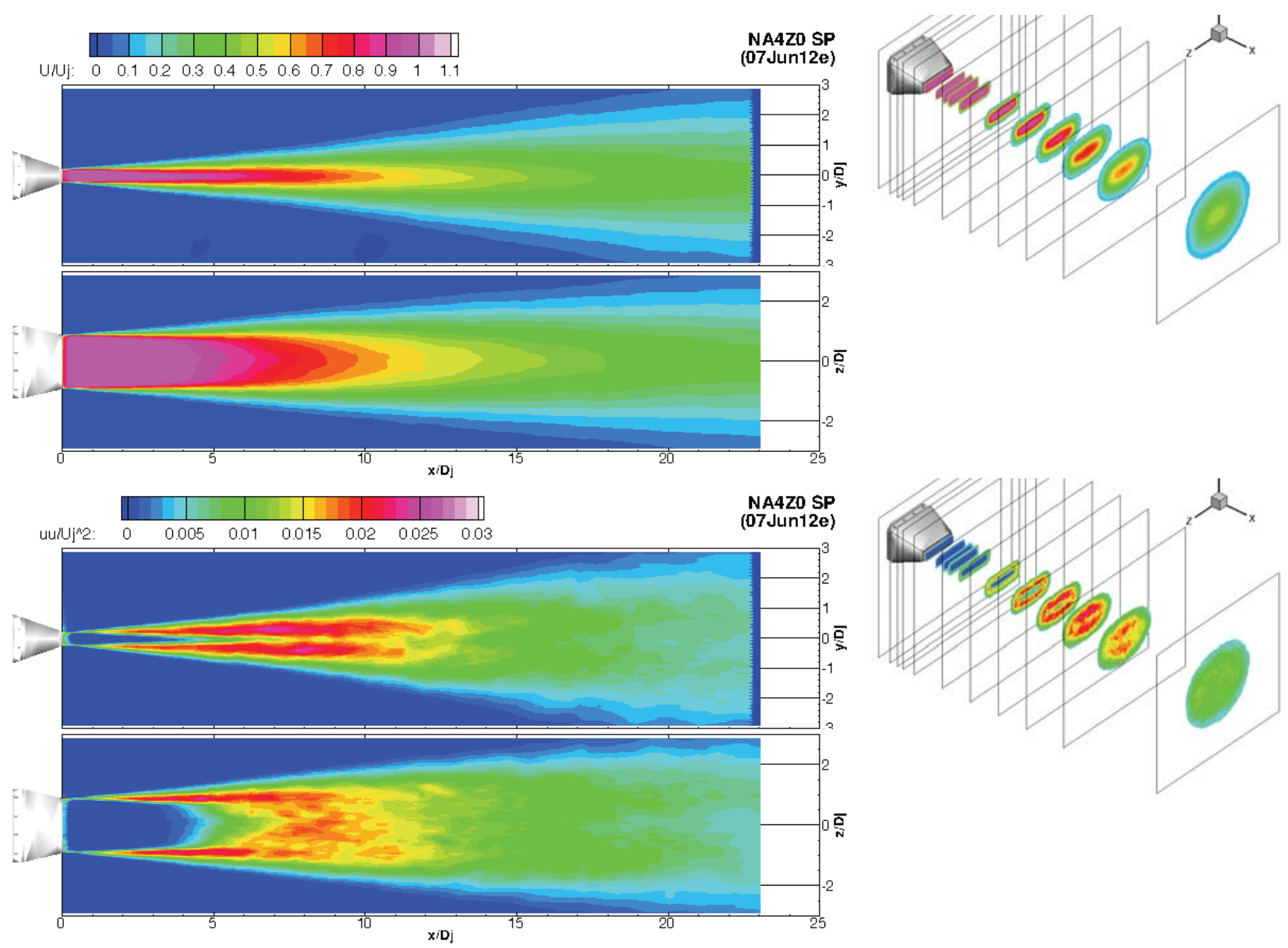

Figure 4 Mean (top) and variance (bottom) of axial velocity from baseline aspect ratio 4 rectangular jet, Ma=0.9, unheated. 


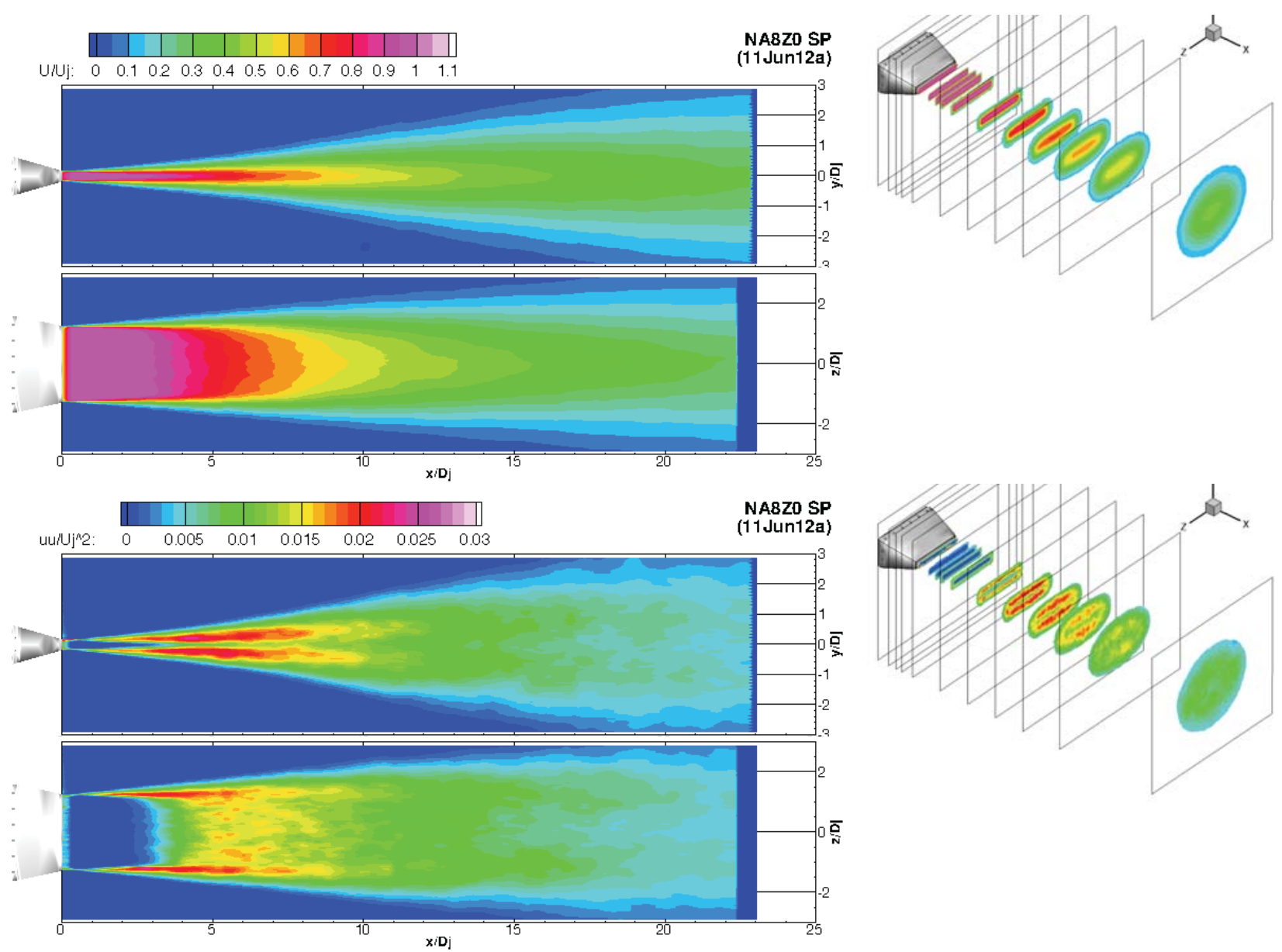

Figure 5 Mean (top) and variance (bottom) of axial velocity from baseline aspect ratio 8 rectangular jet, $M a=0.9$, unheated.

Contour plots serve to deliver overall qualitative understanding of the differences in the nozzle flow fields. However, to directly compare such measures as potential core length, location and amplitude of turbulence line plots are required. In Figure 6 the mean and variance of axial velocity along the centerline and lipline of the round nozzle are plotted versus axial distance. From the centerline profile the potential core length can be picked out as roughly $7.8 D_{j}$, a value in keeping with previous findings ${ }^{8}$. The peak variance of axial velocity is roughly 0.016 , corresponding to an rms value of $u^{\prime} / U_{j}=12.6 \%$, also in line with published data. On the lipline the variance reaches a maximum value of 0.022 , or $u^{\prime} / U_{j}=14.5 \%$, a value that is within experimental error of measurements of round jets. The near-duplicate lines on the lipline plot are from both sides of the nozzle, demonstrating symmetry and experimental error. 

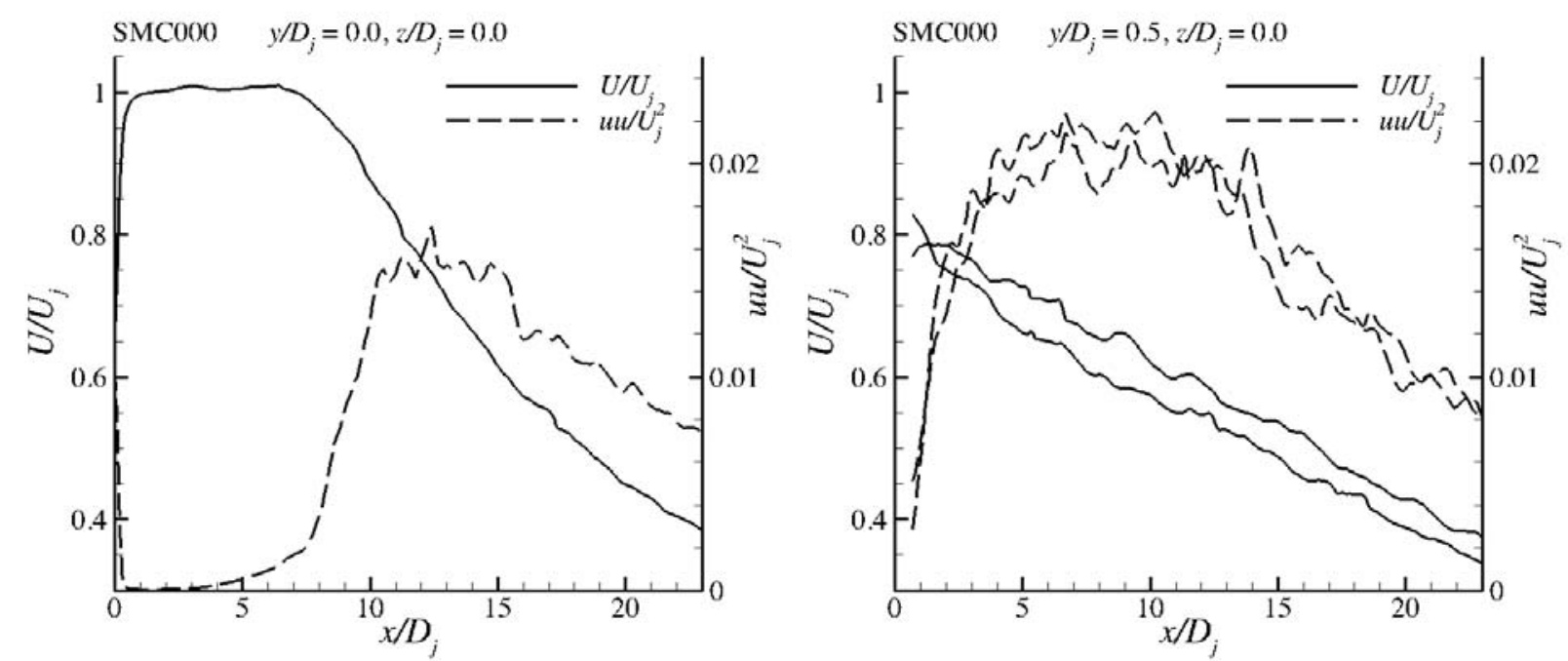

Figure 6 Mean and variance of axial velocity on centerline (left) and on lipline (right) of round nozzle at Ma $=0.9$, unheated.

Similar to the plots of mean and variance of velocity on the centerline and lipline shown above for the round nozzles, data from the baseline rectangular nozzle is shown in Figure 7. In the case of rectangular nozzles there are two planes of symmetry, and the data on both liplines are shown. There are two lines even on the centerline plots because two measurement planes were acquired which intersected on the centerline. The agreement between the two independently measured lines gives a measure of the accuracy of the measurement.

The mean velocity on the centerline gives the first-order impact of aspect ratio-a foreshortened potential core. For an unheated float at $M a=0.9$ the potential core shrank from $x / D_{j}=7.8$ (round jet) to $6.0(2: 1), 4.0(4: 1)$, and $3.0(8: 1)$. If the axial distance was normalized by the minor axis dimension $h$ instead of equivalent diameters then these three values would all become $x / h \sim 8.7$ (see Discussion). However, in this work all lengths will continue to be normalized by equivalent diameter.

The peak values of the axial variance $u u / U_{j}^{2}$ were slightly higher for the higher aspect ratio rectangular nozzles than in the round jet, ranging from $0.016(8: 1)$ to $0.019(2: 1)$ compared to the 0.016 value for the round jet.

Along the liplines, the mean velocity did not show a smoothly decreasing velocity with distance, as did the round jet. Particularly in the 4:1 and 8:1 nozzle flows there is a region just downstream of the end of the potential core where the velocity stops decaying with distance before resuming its decay. Values of peak axial variance ran from $0.028(2: 1)$ to 0.02 (8:1) on the 
minor axes, but were a more consistent 0.022 value on the major axes, roughly the same as in the round jet. The shapes of the turbulent profiles along the liplines were rather different as well. For starters, the profiles of $u u / U_{j}^{2}$ along the liplines of the rectangular nozzles had a rather sharper peak than in the round jet. The locations of these peaks varied on the two axes, being closer to the nozzle on the major axis than on the minor axis. In fact, the peak in the turbulence on the major axis seems to correspond to the end of the potential core.
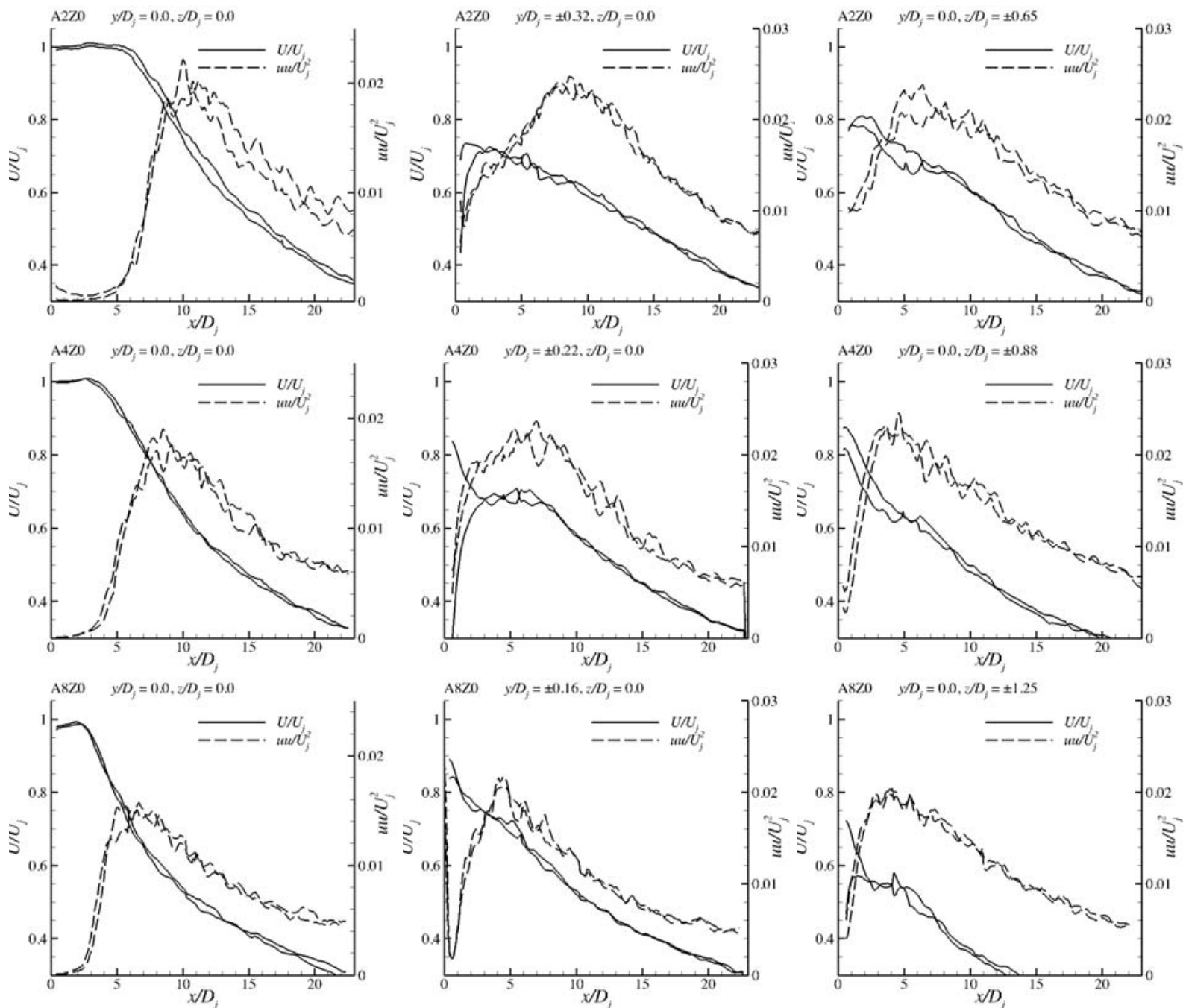

Figure 7 Mean and variance of axial velocity on centerline (left), minor-axis lipline (middle), and majoraxis lipline (right) of rectangular nozzles with aspect ratio 2:1 (top), 4:1 (center), and 8:1 (bottom) at Ma $=0.9$, unheated.

\section{Beveled Rectangular Nozzles}

Turning to the impact of the bevel on rectangular jet plumes, Figure 8 shows an overview of the turbulence from the beveled 8:1 rectangular nozzle. The turbulence in the shear layer downstream of the long side of this nozzle is clearly different from the turbulence in the shear 
layer downstream of the short side of the nozzle. Line plots have been prepared to quantify the impact of having the shear layer start later on one side of the nozzle than the other. Figure 9 relates the mean and variance of axial velocity for the 2:1 rectangular nozzle with two different bevel lengths, $L / D_{j}=0.63$ and $L / D_{j}=1.25$. In the left-hand column, the centerline mean and variance profiles are compared with the basic rectangular nozzle with no bevel. The curves fall on one another within experimental accuracy. Looking at the axial variance of velocity along the short and long liplines in the right-hand column of plots, there is similarly not much impact of bevel length on the flow. Other than a delay in the onset of turbulence near the nozzle on the long side of the nozzle $\left(y / D_{j}=-0.31\right)$, the short bevel nozzle shows no difference from the basic rectangular nozzle. The long bevel nozzle has some increase in turbulence in the initial shear layer on its long side, but is indistinguishable from the short side and basic nozzle by the end of the potential core.

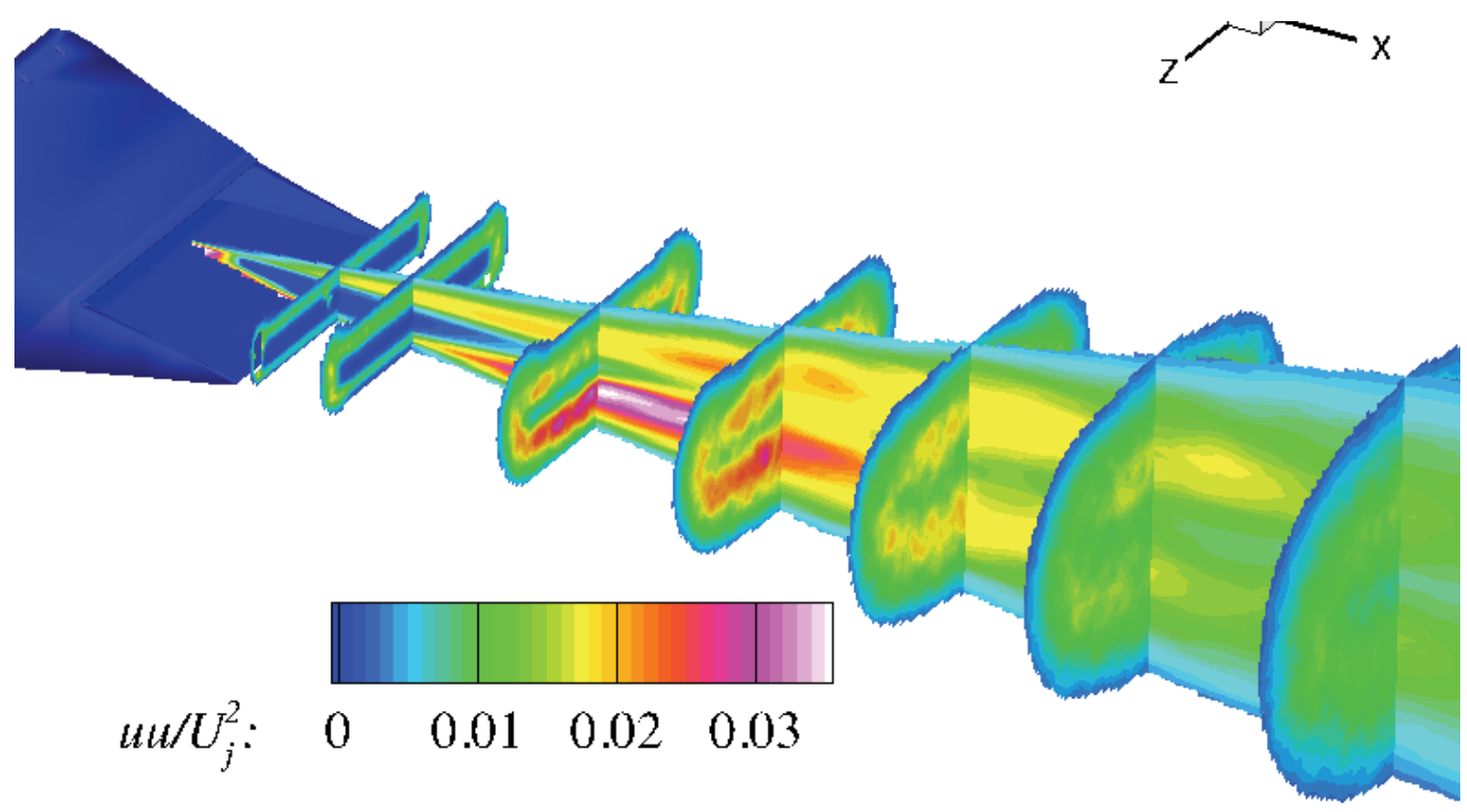

Figure 8 Variance of axial velocity from beveled 8:1 rectangular nozzle at Ma $=0.9$, unheated. 

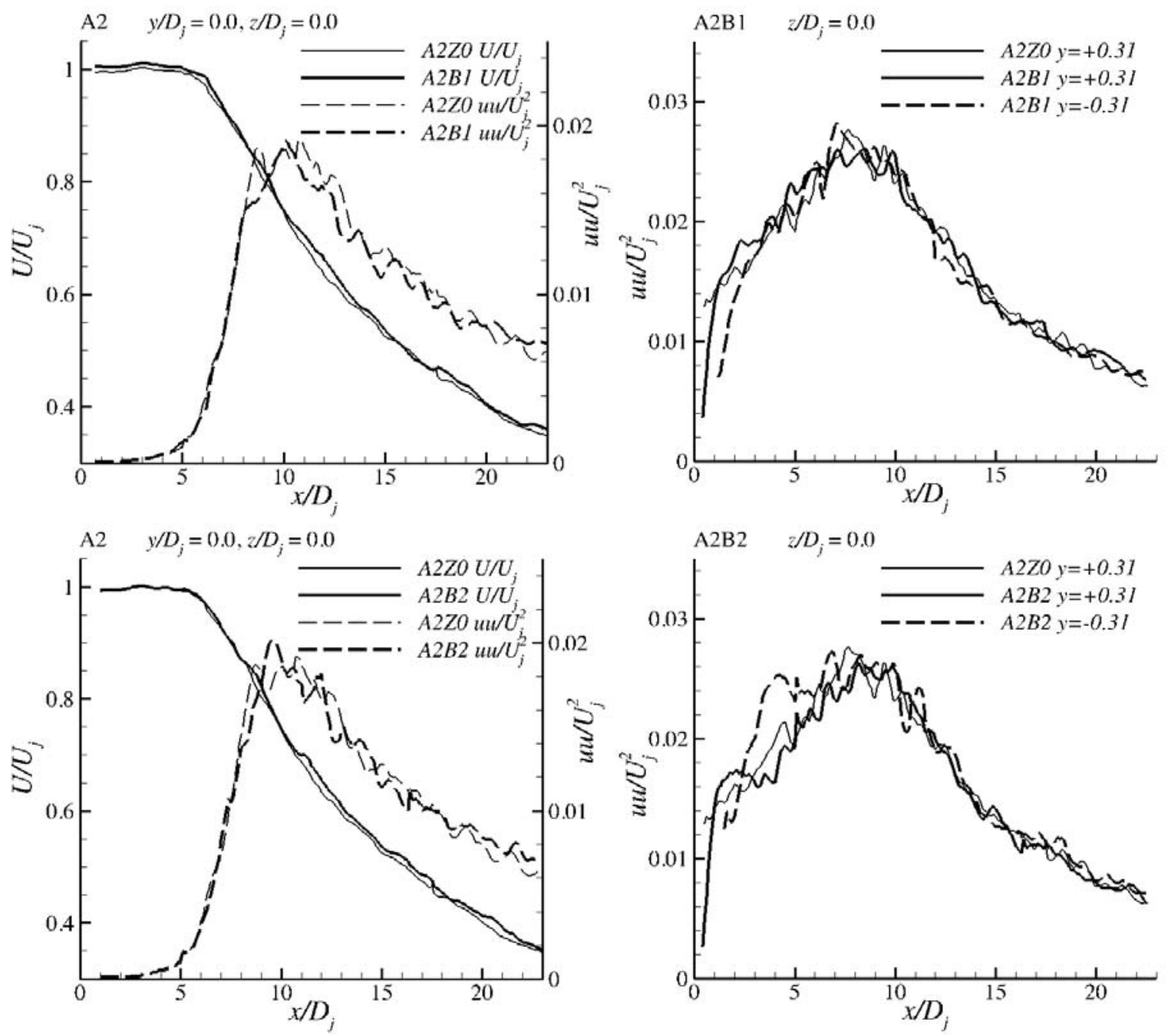

Figure 9 Velocity profiles in 2:1 rectangular jet with bevel length $L / D_{j}=0.63$ (top) and $L / D_{j}=1.25$ (bottom). Centerline profiles of $U / U_{j}$ and $u u / U_{j}^{2}$ of axial velocity (left) and lipline profiles of $u u / U_{j}^{2}$ (right) from short and long side of beveled nozzles. Basic 2:1 rectangular nozzle data in light lines for reference. $M a=0.9$, unheated

The 4:1 rectangular nozzle with bevel is examined next (Figure 10). The centerline profiles of $U / U_{j}$ and $u u / U_{j}^{2}$ show little difference from the basic rectangular nozzle. The turbulence on the liplines, however, does show an impact. On the long side of the bevel the shear layer quickly grows in turbulence for both the long and short bevel, surpassing the basic and short side turbulence levels in the region $2<x / D_{j}<8$. This increase in turbulence is accentuated by increasing the length of the bevel. 

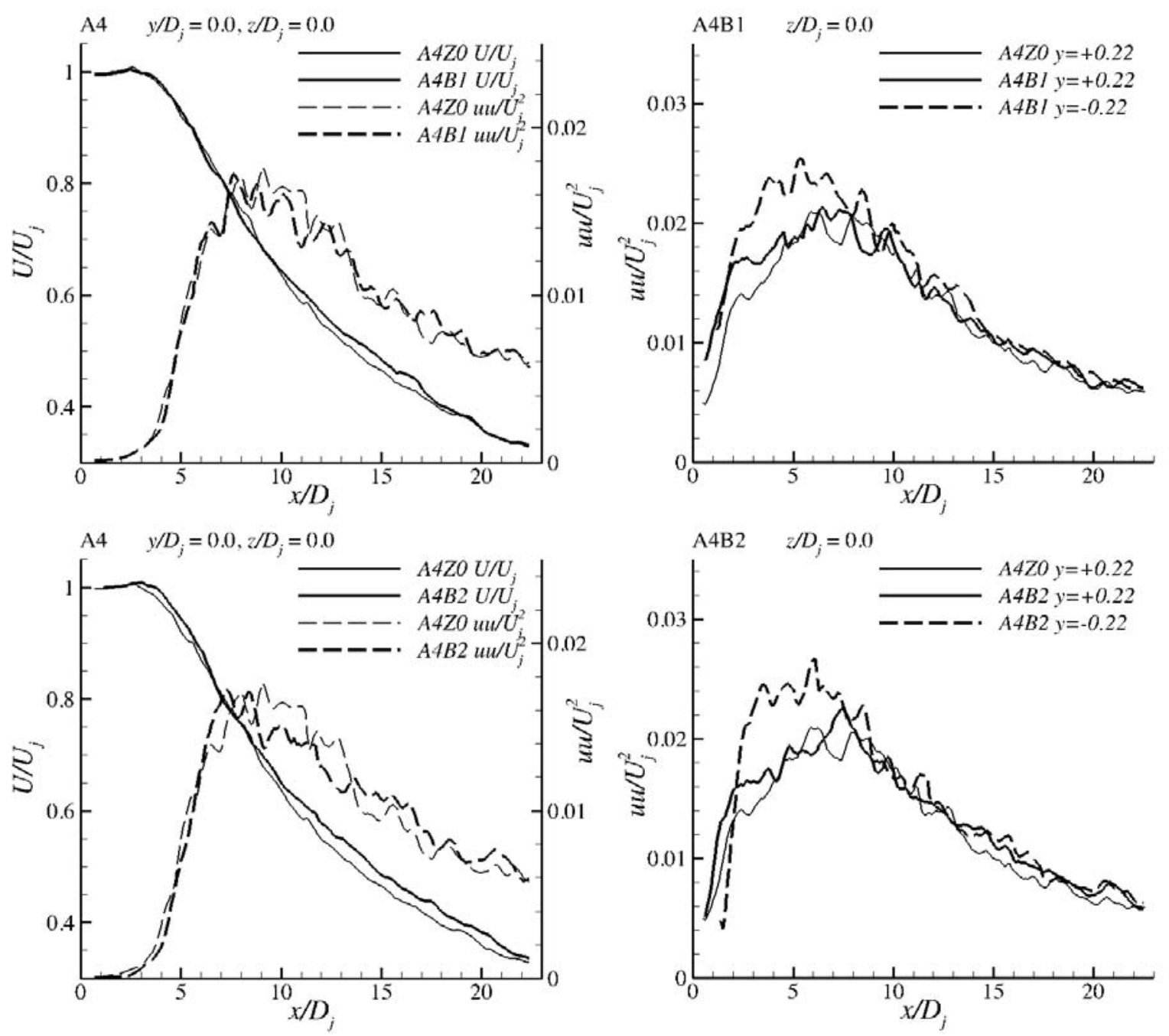

Figure 10 Velocity profiles in 4:1 rectangular jet with bevel length $L / D_{j}=0.63$ (top) and $L / D_{j}=1.25$ (bottom). Centerline profiles of $U / U_{j}$ and $u u / U_{j}^{2}$ of axial velocity (left) and lipline profiles of $u u / U_{j}^{2}$ (right) from short and long side of beveled nozzles. Basic 4:1 rectangular nozzle data in light lines for reference. $M a=0.9$, unheated

The highest aspect ratio nozzles, with aspect ratio 8:1, do show an impact of bevel length, even on the centerline of the jet. In Figure 11 the mean velocity along the jet centerlines is not affected by the bevel length, but the turbulence $\left(u u / U_{j}^{2}\right)$ is, peaking sooner and higher than in the corresponding basic 8:1 nozzle flow. The impact of the bevel is even more extreme on the liplines. The peak variance of axial velocity is $30 \%$ higher on the lipline of the long side of the short bevel relative to the short side and to the basic rectangular nozzle. It is $75 \%$ higher for the long bevel nozzle. 

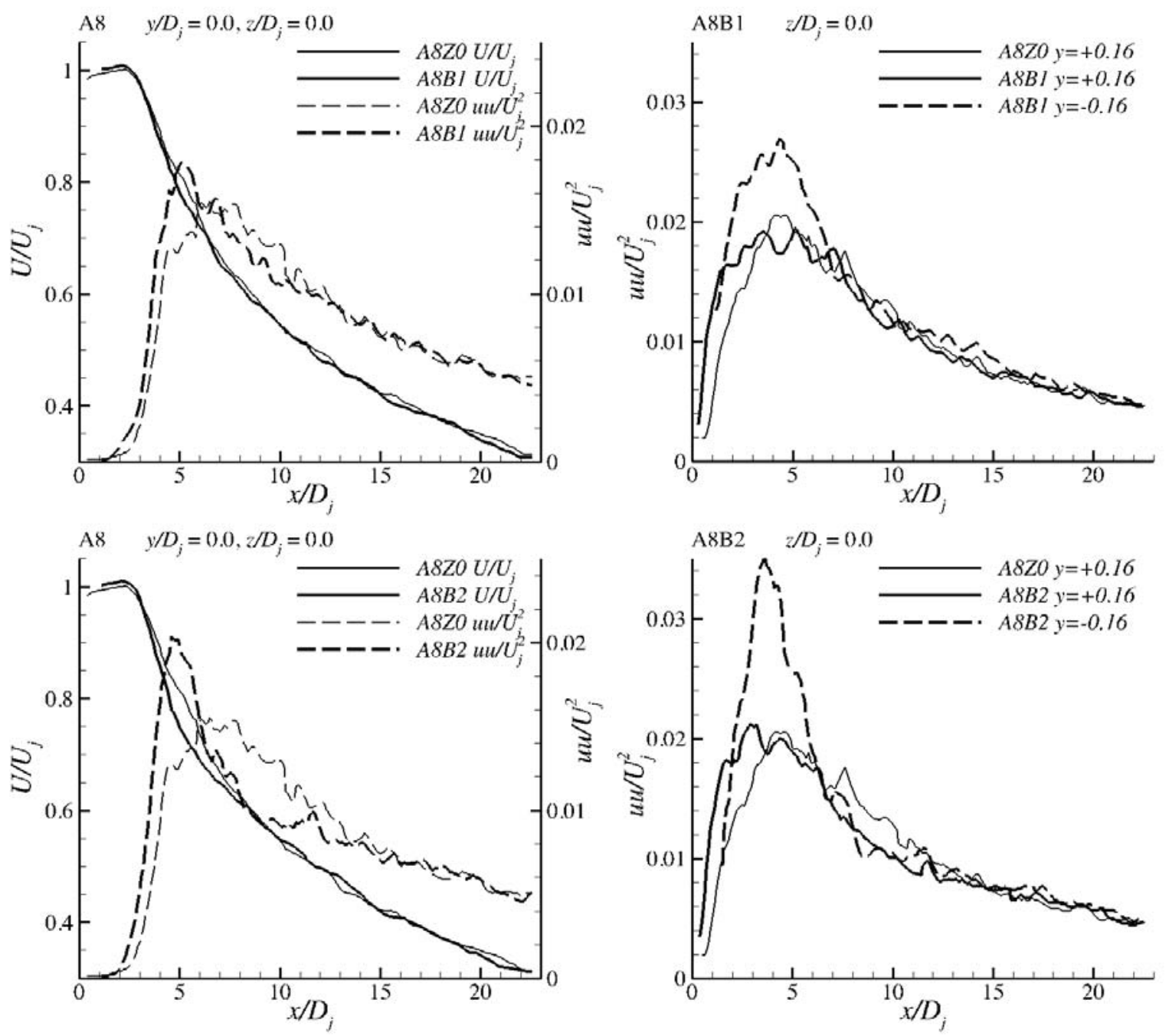

Figure 11 Velocity profiles in 8:1 rectangular jet with bevel length $L / D_{j}=0.63$ (top) and $L / D_{j}=1.25$ (bottom). Centerline profiles of $U / U_{j}$ and $u u / U_{j}^{2}$ of axial velocity (left) and lipline profiles of $u u / U_{j}^{2}$ (right) from short and long side of beveled nozzles. Basic 8:1 rectangular nozzle data in light lines for reference. $M a=0.9$, unheated

\section{B. Anisotropy of turbulent velocity}

One of the purposes of this report is to provide validation data for comparison with ReynoldsAveraged Navier-Stokes (RANS) solutions, hence we address the relationship between the components of turbulent velocity and the turbulence kinetic energy (TKE). By definition TKE is the sum of the variance of orthogonal velocity components $u, v, w$ :

$$
T K E=1 / 2(u u+v v+w w) .
$$

The nomenclature of 'components of TKE' comes readily by splitting out this sum. In measurements it is often the case that only one or two of the velocity components can be measured and approximations between the measured component and the full TKE are useful. 
The relationships between the components are also of importance in models of acoustic source strength. These relationships will be considered next.

Define the total cross-stream turbulent energy $q q$ as the sum of the two cross-stream components,

$$
q q=v v+w w=r r+t t
$$

where $v v, w w$ are the cross-stream components in Cartesian coordinates and $r r, t t$ are the radial and tangential components in a cylindrical coordinate system. In the round jet, it has been noted that the axial component of turbulent velocity $u и$ is larger than the cross-stream components and that the tangential component $t t$ is slightly larger than the radial component $r r$. To visualize this the combined magnitude of the two cross-stream components $q q$ is compared with the axial component $u u$ by making the ratio $q q / u u$. If the measurements are without directional bias, and the jet is experimentally clean, then this field should be axisymmetric. Secondly, to quickly check for directional bias in the measurement and to find the relationship between $r r$ and $t t$, the ratio $r r / t t(=v v / w w$ along $y)$ is plotted for the cross-stream planes.

Figure 12 shows $q q / u u$ plotted for all the cross-stream planes for regions where the axial turbulence intensity $u и$ is greater than 0.002 , or roughly $10 \%$ of its peak value. Visually, the disparities from uniformity have no pattern associated with the quadrants of the jet and appear random. There is also little radial difference over the region where turbulence is significant, except perhaps on the inner edge of the shear layer in the potential core. Furthermore, $q q / u u$ seems to average around 1 over the potential core, increasing with axial distance.

To figure out how this cross-stream turbulent kinetic energy $q q$ is split between the transverse $t t$ and radial $r r$ components, the ratio of vertical and horizontal components, $v v / w w$, is analyzed. Again, for an axisymmetric jet this pattern should exactly produce a four-quadrant pattern with the $45^{\circ}$ lines being exactly equal to one and the values on the primary axes being either $r r / t t$ (along the $y$-axis) or $t t / r r$ (along the $z$-axis). From the figure (note the exponential color scale), the value for $r r / t t$ seems to be roughly 1.2, slightly decreasing with downstream distance. From these values it is estimated that the ratios of turbulent velocity variances uu:rr:tt are 1:0.55:0.65. Subsequent analysis of the subsonic hot jet case found this to hold as well. However, this ratio did not hold in the case of supersonic cases (not shown here), both ideally expanded and underexpanded. More test cases will be required to understand this observation. 


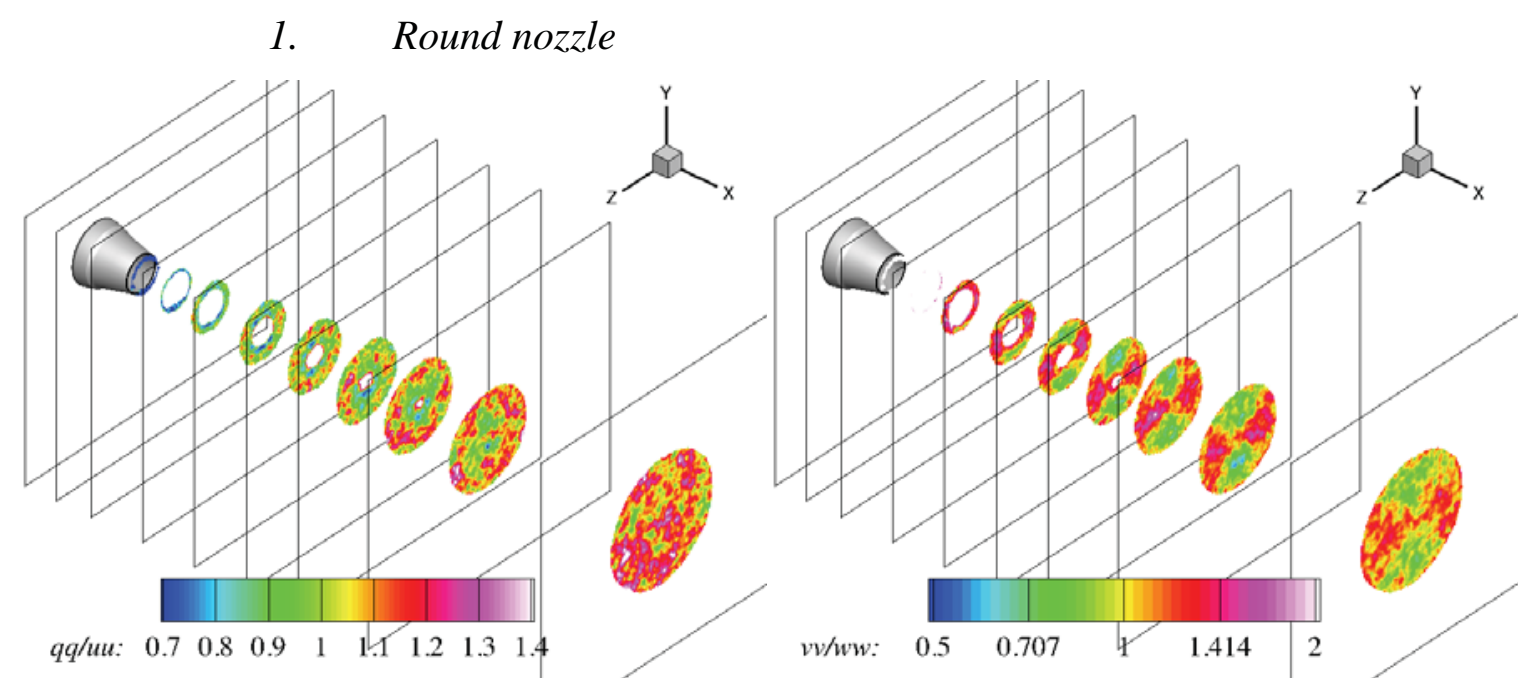

Figure 12 Anisotropy of turbulence: cross-stream relative to axial (left); vertical relative to horizontal (right). Round nozzle.

\section{Rectangular nozzles}

The same analysis of the turbulent velocity components was carried out on the rectangular nozzles. These are a bit more complicated in that the shear layer does not lie on a constant radial coordinate and thus transverse and radial components are not as useful in describing the shear layer.

In Figure 13 the ratio of cross-stream to axial components of TKE are shown for three baseline rectangular nozzles. The first observation is that there appears to be more systematic variation in $q q / u u$ for the rectangular nozzles than for the round nozzle. The ratio is consistently lower on the high-speed side of the shear layer, more pronounced than in the round jet. Also like the round jet the average ratio increases with downstream distance, but the ratio is higher for the same downstream locations (same distance in equivalent diameters). Furthermore this trend increases with increasing aspect ratio.

The plots of the ratio $v v / w w$ require more thought than in the case of the round jet. In the potential core region where there are holes in the contour plots because there is no turbulence the ratio has similar levels as the round jet on the primary axes. Values in the ratio are 1.4 or slightly more on the $z$-axis and the inverse on the $y$-axis. Indeed along the potential core the shear layer is very two-dimensional on each side of the nozzle and the anisotropy is similar along the shear layers. After the end of the potential core the ratio is greater than 1 near the centerline. This is consistent with a predominant flapping of the jet in the minor axis plane. By the last 
measurement station the cross-section has become round and $v v / w w$ ratio distribution becomes similar to that of the round jet.

In an attempt to better quantify the trends described above for the ratio of cross-stream to axial components $q q / u u$, the values of $q q / u u$ have been averaged over the measurement planes for the regions shown (e.g. where $u u / U_{j}^{2}>0.002$ ). The spatial average $\langle q q / u u\rangle$ as a function of downstream distance is plotted in Figure 14 for all three baseline rectangular nozzles and the axisymmetric SMC000 nozzle. 


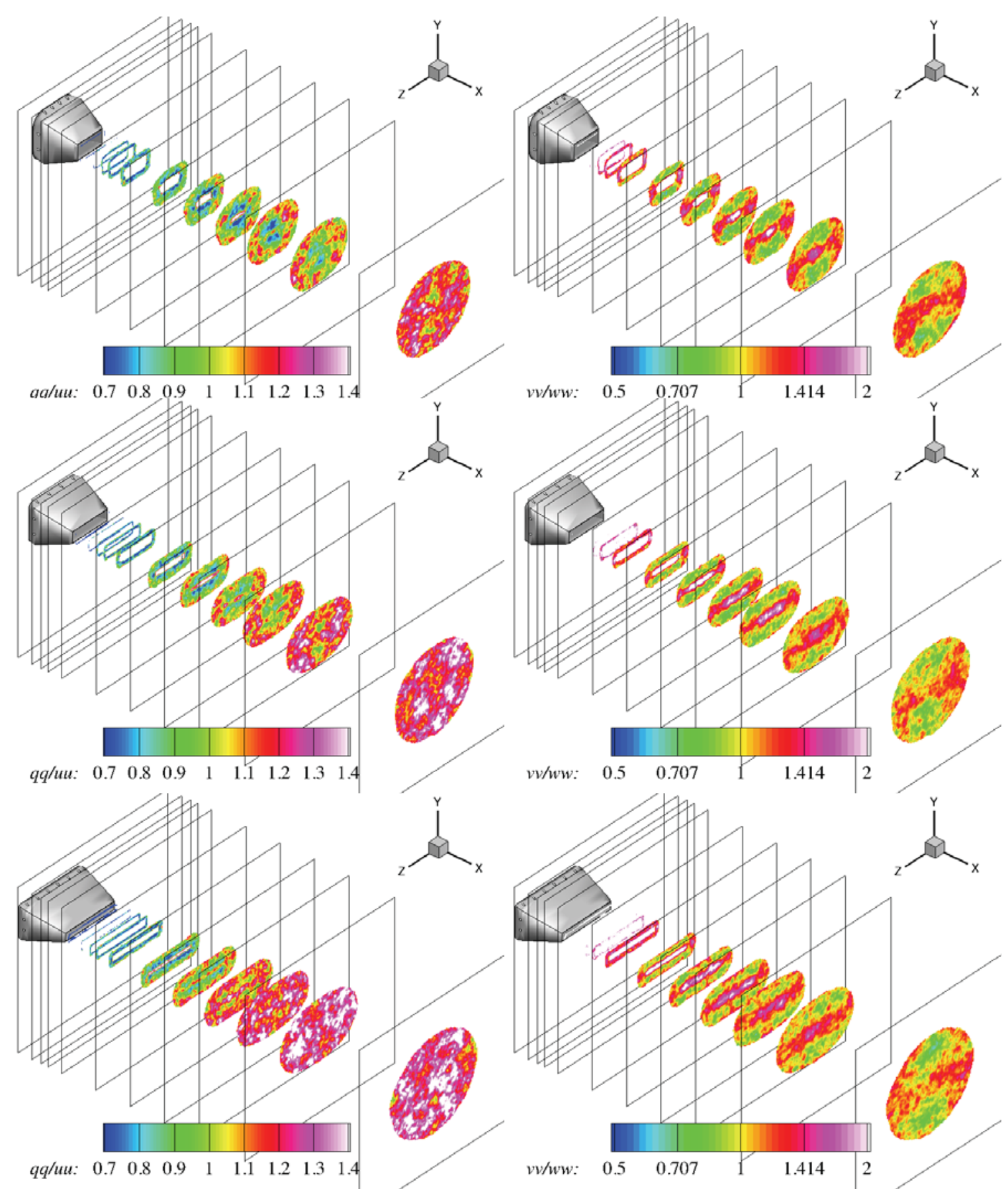

Figure 13 Anisotropy of turbulence: transverse relative to axial (left); vertical relative to horizontal (right). Aspect ratio of rectangular nozzle: 2:1 (top), 4:1 (middle), 8:1 (bottom).

As described above, the round jets have an average value of $q q / u u$ between 1 and 1.1 over the range of reliable measurements (measurements made upstream of $x / D_{j}=3$ showed some disagreement between the streamwise and cross-stream measurements and were excluded here). The 2:1 aspect ratio nozzle has essentially the same value for $\langle q q / u u\rangle$. However, the 4:1 and 8:1 do show a significant increase in the importance of cross-stream turbulence. 


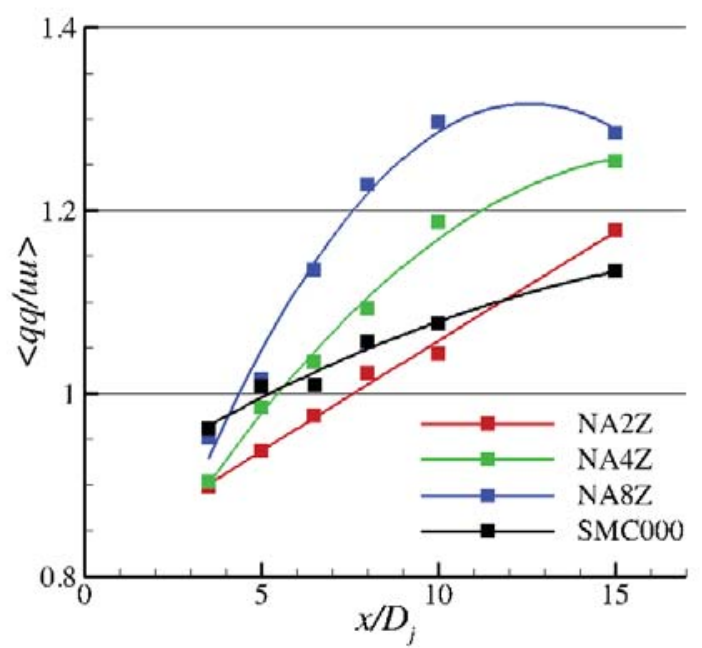

Figure 14 Ratio of cross-stream to axial components of turbulent kinetic energy for basic rectangular nozzles and round nozzles, plotted against axial distance normalized by equivalent diameter.

Adding bevel to the rectangular nozzles (adding length to one wide side of the nozzle) did not greatly affect the turbulence statistics shown here. This includes the ratio of cross-stream to axial turbulence as shown in the figure below. In each plot of Figure 15 a different aspect ratio nozzle family is shown with the different bevel lengths compared in different line styles. In general the addition of the bevel reduces the cross-stream turbulence amplitudes relative to the axial component after the end of the potential core.
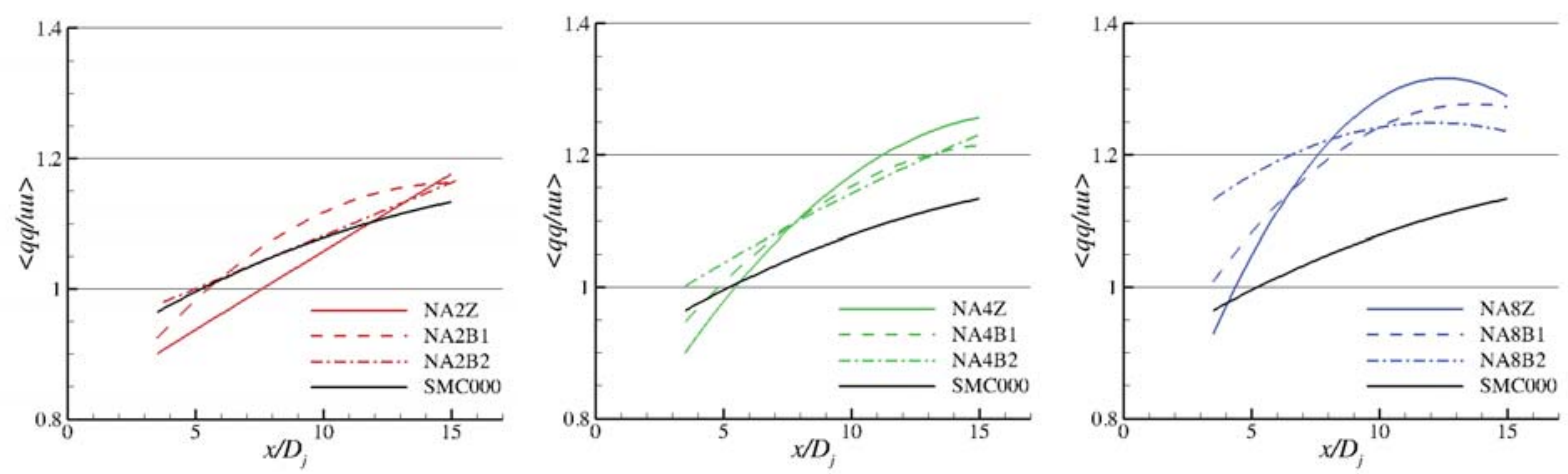

Figure 15 Ratio of cross-stream to axial components of turbulent kinetic energy for beveled nozzles with aspect ratios 2:1 (left), 4:1 (center), and 8:1 (right), plotted against axial distance normalized by equivalent diameter $D_{j}$.

\section{Lengthscales of Turbulence}

Beyond modeling the strength of acoustic sources using turbulent kinetic energy from RANS solutions, acoustic analogy codes also require the dissipation fields to estimate time and lengthscales. A lengthscale can be estimated from spatial correlations of velocity $R_{i j}$ using the 
PIV data. For clarity, indices 1,2,3 will be used in place of $u, v, w$ and $x, y, z$ in the notation of the lengthscales.

$$
\begin{aligned}
& R_{i j}(\mathbf{x}, \xi)=\frac{1}{N U^{2}} \sum_{N} u_{i}(\mathbf{x}) u_{j}(\mathbf{x}-\xi) \\
& L_{i j}(\mathbf{x})=\frac{1}{D} \int_{-\infty}^{\infty} R_{i i}(\mathbf{x}, \xi) d \xi_{j}
\end{aligned}
$$

Note that several lengthscales are possible depending upon choice of velocity component and spatial direction. The lengthscales presented here are defined by the spatial separation where $R_{\mathrm{ij}}$ matches $1 / \mathrm{e}$, as adopted by references 10 and 11 . The 1/e definition focuses on the initial decay of the correlations and implicitly assumes a Gaussian functional form for the correlation. These lengthscales were found to be very similar to the conventional integral lengthscale given in equation (4), but the 1/e definition is more robust, eliminating the uncertainty from integrating the noisy tails and from the finite basis of the correlations.

Looking first at the longitudinal axial lengthscale $L_{11}$ in Figure 16, note that the lengthscale varies from 0.1 to 2 . The color contours are spaced logarithmically to accentuate the changes at the small scales near the nozzle. The black contour outlines the region within which the TKE is roughly half of its peak, discerning what regions are critical for turbulence modeling. Lengthscales within the potential core, given by the inner wedge of the TKE contour, and well outside the TKE contour, are not meaningful as there is no turbulence there.

Batchelor ${ }^{12}$ showed that the longitudinal lengthscale $L_{i i}$ is related to the lateral lengthscale $L_{i j}$ $(i \neq j)$ by $L_{i i}=2 L_{i j}$ in isotropic turbulence. Similar relations are often used in acoustic source modeling even though the turbulence is obviously not isotropic nor homogeneous. In Figure 16 these relationships are documented. Comparing $L_{11}$ with $L_{12}$ in the round nozzle data, the color contours are nearly equivalent after the $L_{12}$ color scale is adjusted by a factor of 2 . In the other lateral relationship, $L_{22}$ is compared with $L_{21}$ in Figure 17. Here the two plots are similar when the lateral lengthscale contours are adjusted by a factor of 1.4. Comparing across figures, the longitudinal $L_{11}$ lengthscale is roughly twice the longitudinal $L_{22}$ lengthscale in magnitudeclearly not isotropic. Apart from the differences in magnitude, there is a perceptive difference in the distribution of correlations of axial velocity $\left(L_{11}\right.$ and $L_{12}$; Figure 16$)$ and those of radial velocity ( $L_{21}$ and $L_{22} ;$ Figure 17). In the correlations of axial velocity the lengthscales are much 
shorter near the centerline than on the outer regions of the plume. In the correlations of radial velocity the lengthscales are more nearly uniform across the plume.

\section{Round nozzle}

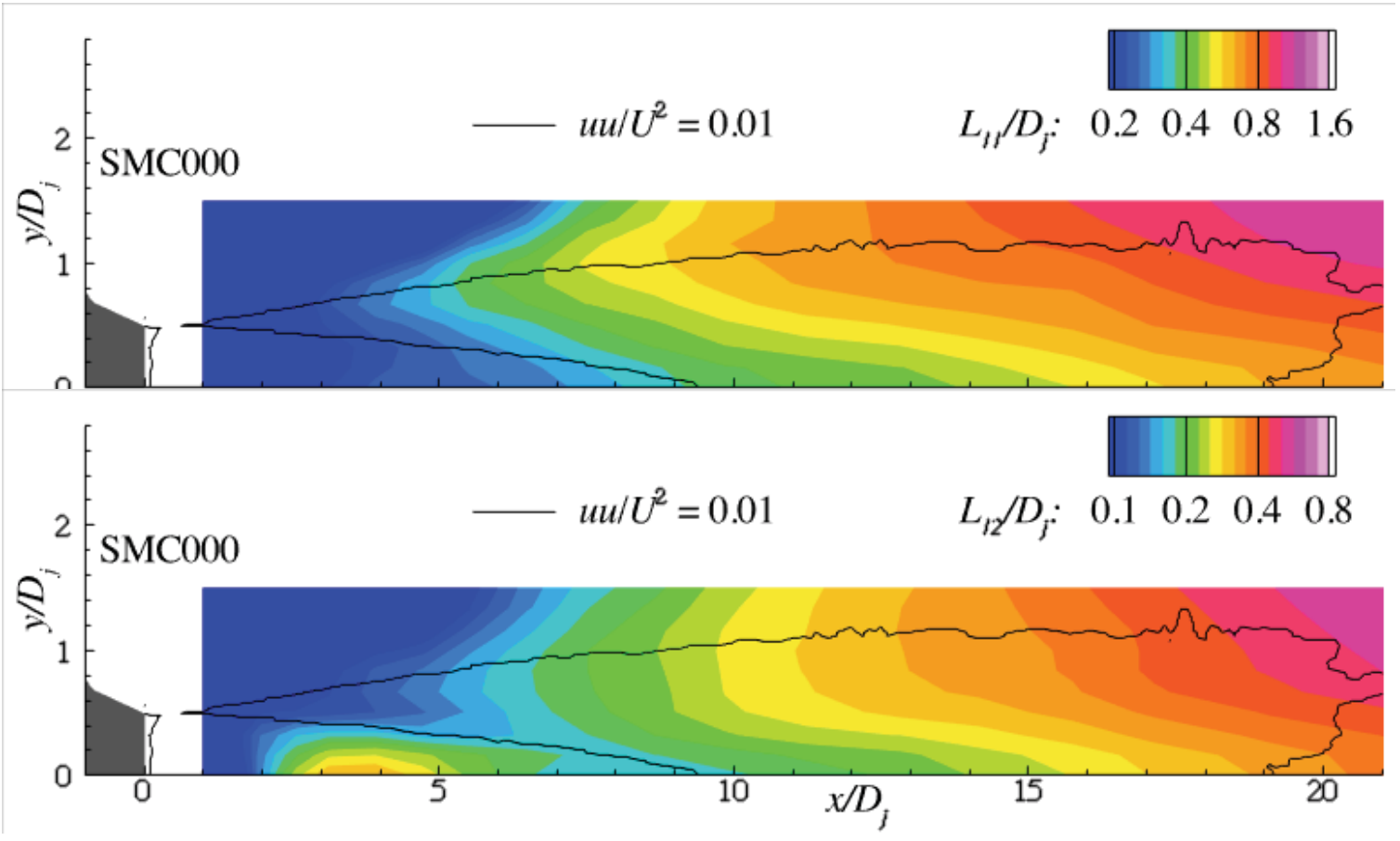

Figure 16 Contour plots of lengthscales in round nozzle, $M a=0.9$, unheated. Longitudinal lengthscale $L_{11}$ (top) and lateral lengthscale $L_{12}$ (bottom). Note logarithmic color scale changed by factor of 2 in lower plot. Black contour line is $u u / U_{j}^{2}=0.01$ for reference. 


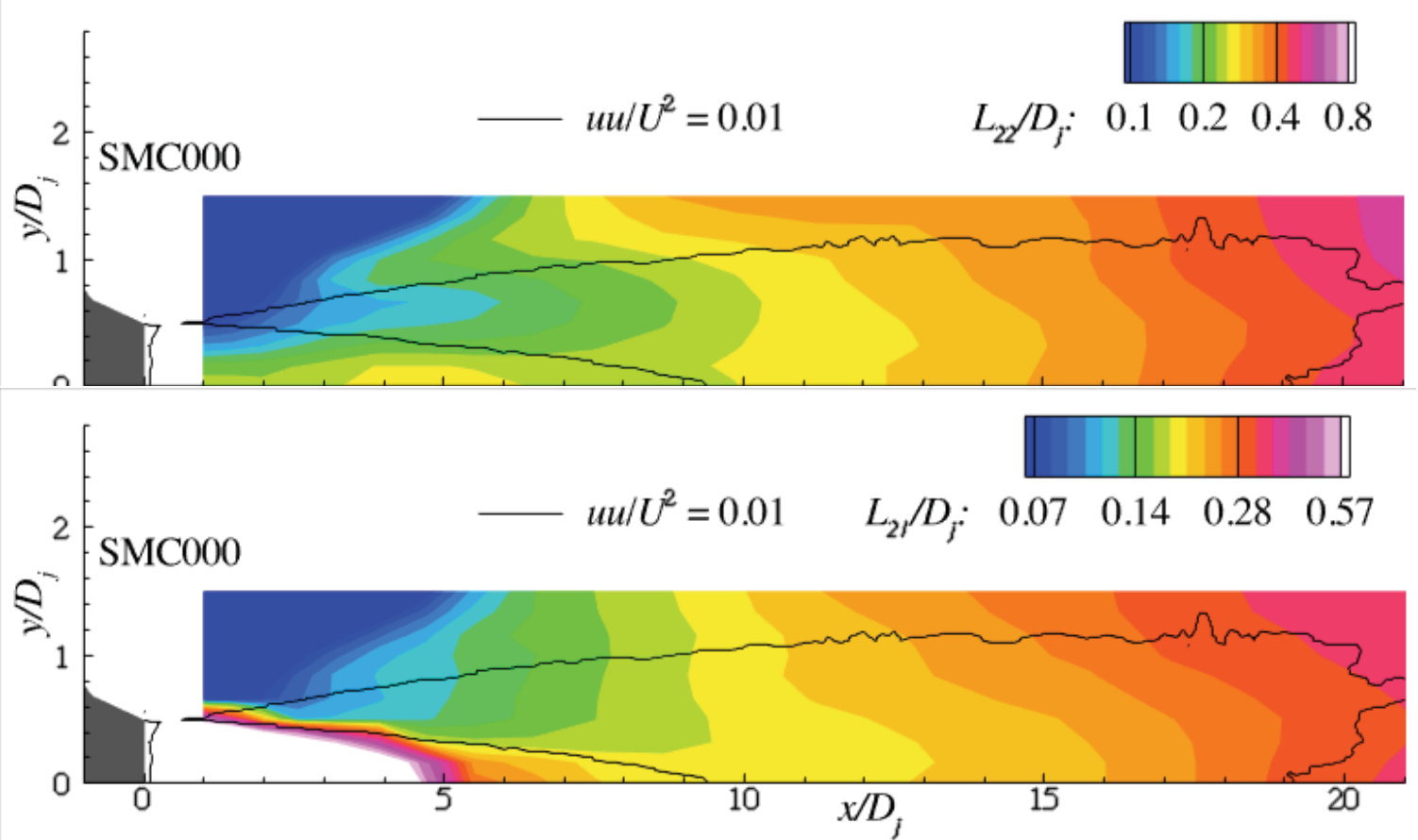

Figure 17 Contour plots of lengthscales in round nozzle, Ma =0.9, unheated. Longitudinal lengthscale $L_{22}$ (top) and lateral lengthscale $L_{21}$ (bottom). Note logarithmic color scale changed by factor of $1.4 \mathrm{in}$ lower plot. Black contour line is $u и / U_{j}^{2}=0.01$ for reference.

\section{Rectangular nozzles}

Modeling the lengthscales in a rectangular jet becomes more complicated by the lack of axisymmetry. Using the streamwise PIV data, the relationship between the lengthscales is explored below. First, the longitudinal axial lengthscale $L_{11}$ is shown for both planes of symmetry in all three basic rectangular nozzles (Figure 18). The increase in $L_{11}$ lengthscale with downstream distance changes with aspect ratio in much the same manner as the potential core and turbulence distributions. The corresponding lateral lengthscales $L_{12}$ (in x,y plane) and $L_{13}$ (in $\mathrm{x}, \mathrm{z}$ plane) do mirror the longitudinal lengthscale at a ratio of roughly 2:1 as attested to by the color contours adjusted by a factor of 2 between Figure 18 and Figure 19. 


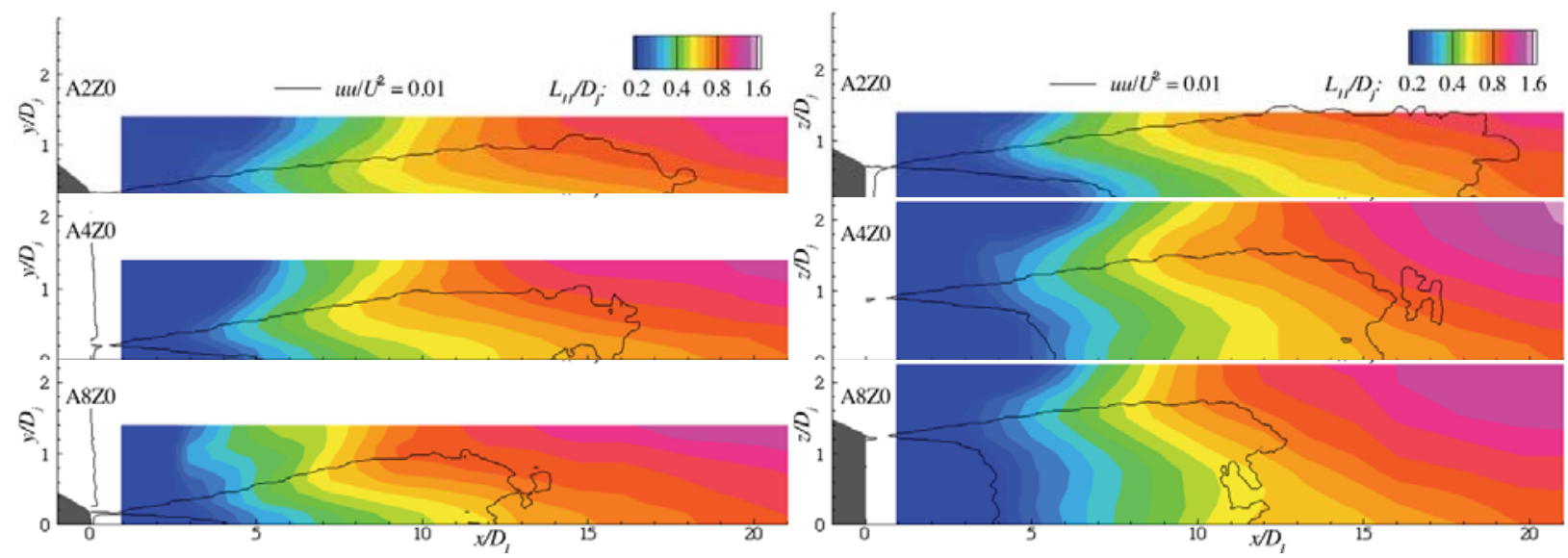

Figure 18 Color contours of longitudinal axial lengthscale $L_{11}$ for rectangular nozzles, aspect ratio 2:1 (top), 4:1 (middle), 8:1 (bottom). Ma $=0.9$, unheated. Black contour line is $u u / U_{j}^{2}=0.01$ for reference.

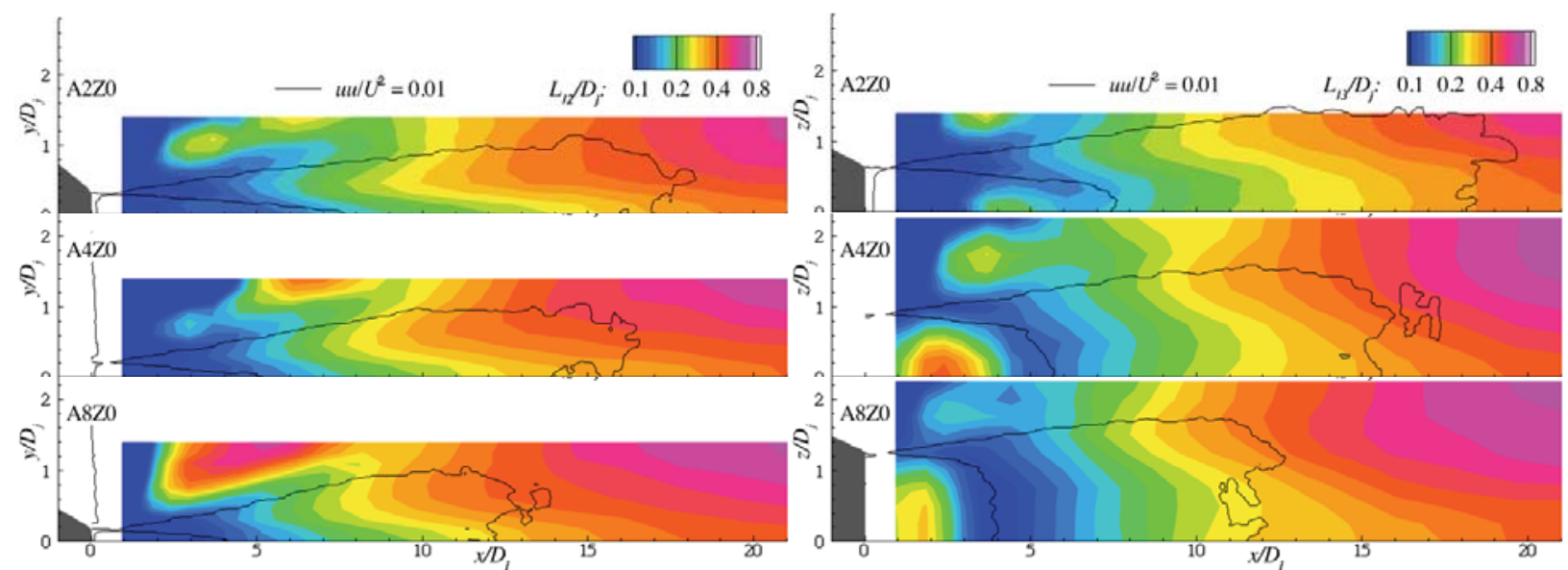

Figure 19 Color contours of lateral lengthscales $L_{12}$ in $(x, y)$ plane (left) and $L_{13}$ in $(x, z)$ plane (right) for rectangular nozzles, aspect ratio 2:1 (top), 4:1 (middle), 8:1 (bottom). Ma =0.9, unheated. Black contour line is $u u / U_{j}^{2}=0.01$ for reference.

The most dramatic effect of non-axisymmetry is found in the cross-stream longitudinal lengthscales, e.g. $L_{22}$ and $L_{33}$ (Figure 20). The longitudinal lengthscales normal to the minor axis are much larger than those in the major axis direction. Just aft of the potential core, the lengthscales in the minor axis are roughly one equivalent diameter. The fact that the jet is correlated over a region larger than the jet width is supportive of the idea that the jet is essentially flapping across the minor axis, strongly modifying the acoustic source in this plane. 


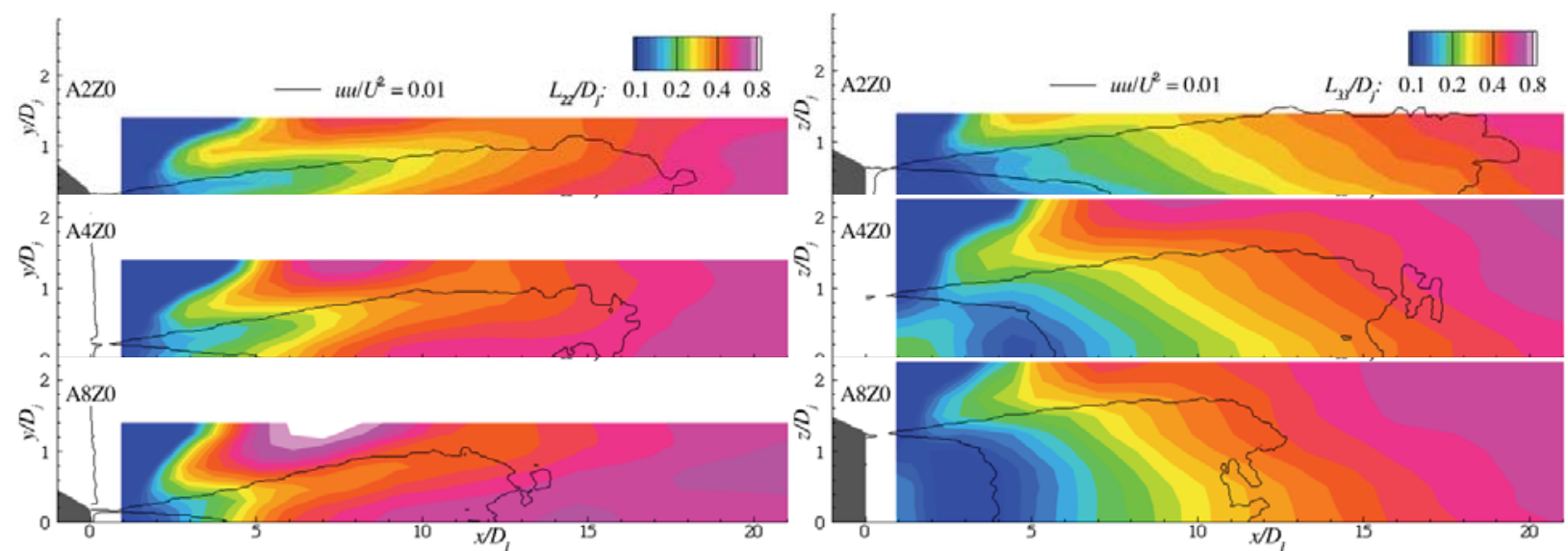

Figure 20 Color contours (log scale) of $L_{22}$ (left), and $L_{33}$ (right) for rectangular nozzles, aspect ratio 2:1 (top), 4:1 (middle), 8:1 (bottom). Ma =0.9, unheated. Black contour line is $u u / U_{j}^{2}=0.01$ for reference.

To quantify trends noted in contour plots, the values of the axial longitudinal lengthscale $L_{11}$ along the jet centerlines are presented in Figure 22. In the plot of $L_{11} / D_{j} v s x / D_{j}$ there are secondorder polynomials fitted through the lengthscale data to show the general trend that lengthscales grow more quickly with increased aspect ratio.

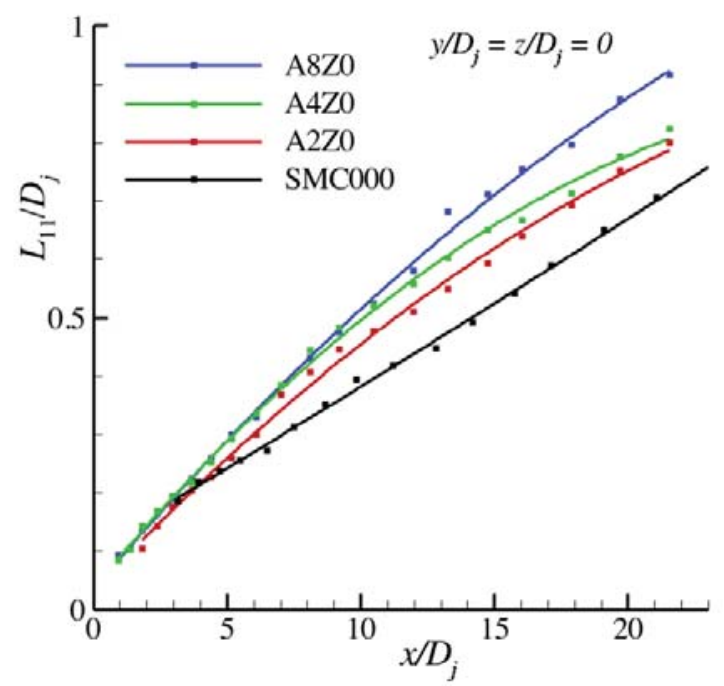

Figure 21 Longitudinal lengthscales $L_{11}$ along centerline of three rectangular jets and a round jet. Ma= 0.9, unheated flow.

To verify the degree to which the lateral and longitudinal lengthscales are related, $L_{11}$ is plotted against $L_{21}$ and $L_{31}$ in Figure 22. The longitudinal lengthscales are given by the left-hand axis while the lateral lengthscales are given by the right-hand axis. The curves bear out the roughly 2:1 scaling factor for longitudinal:lateral lengthscales. 


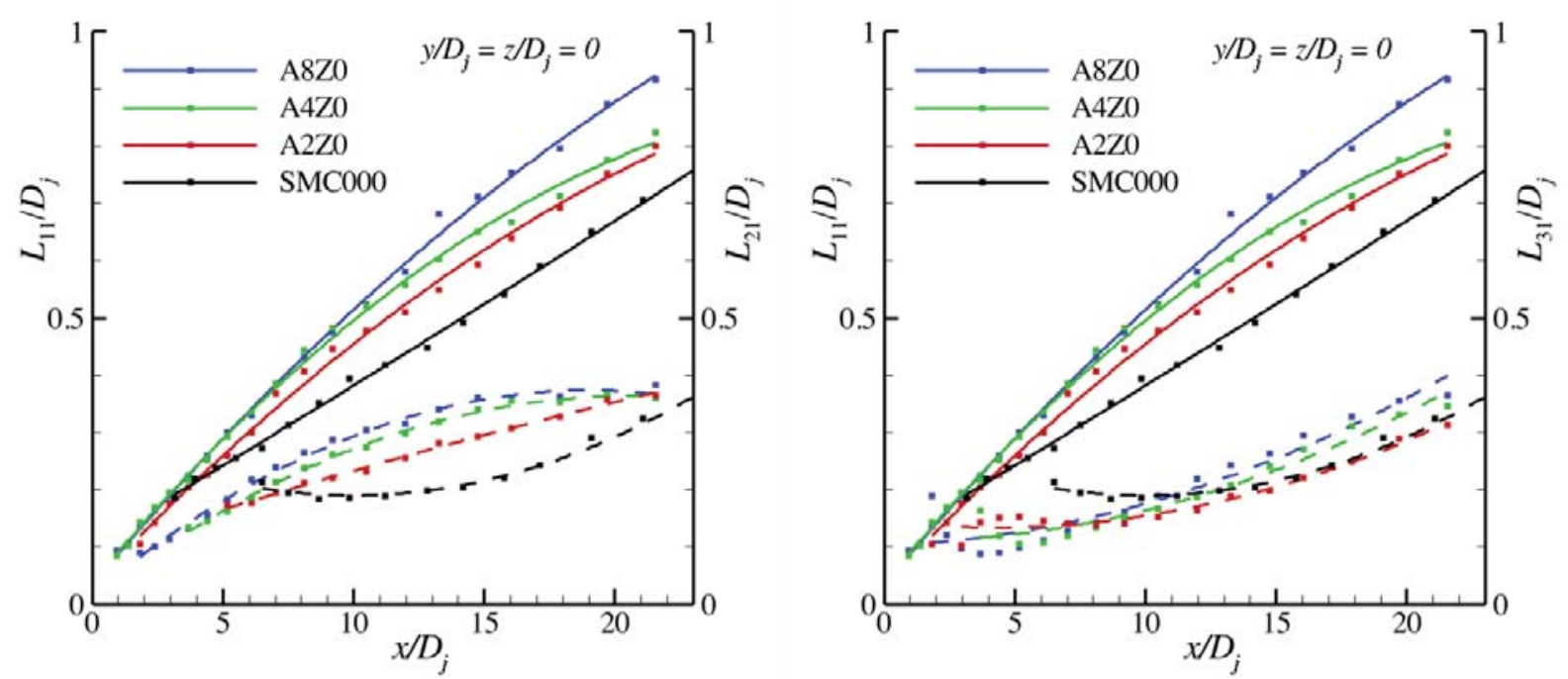

Figure 22 Comparison of longitudinal $L_{11}$ lengthscales (solid lines) with lateral $L_{21}$ (left) and lateral $L_{31}$ (right) lengthscales (dashed lines) along jet centerlines. Ma $=0.9$, unheated flow.

Finally, the cross-stream lateral lengthscales are extracted along the jet centerlines in Figure 23. As remarked above, the $L_{22}$ lengthscale, aligned with the minor axes of the rectangular nozzles, shows a very significant increase over the round jet. The $L_{33}$ lengthscale does not. Clearly there is a very significant large-scale unsteady motion in the plane of the minor axis of the rectangular jets, particularly as the aspect ratio exceeds $2: 1$.
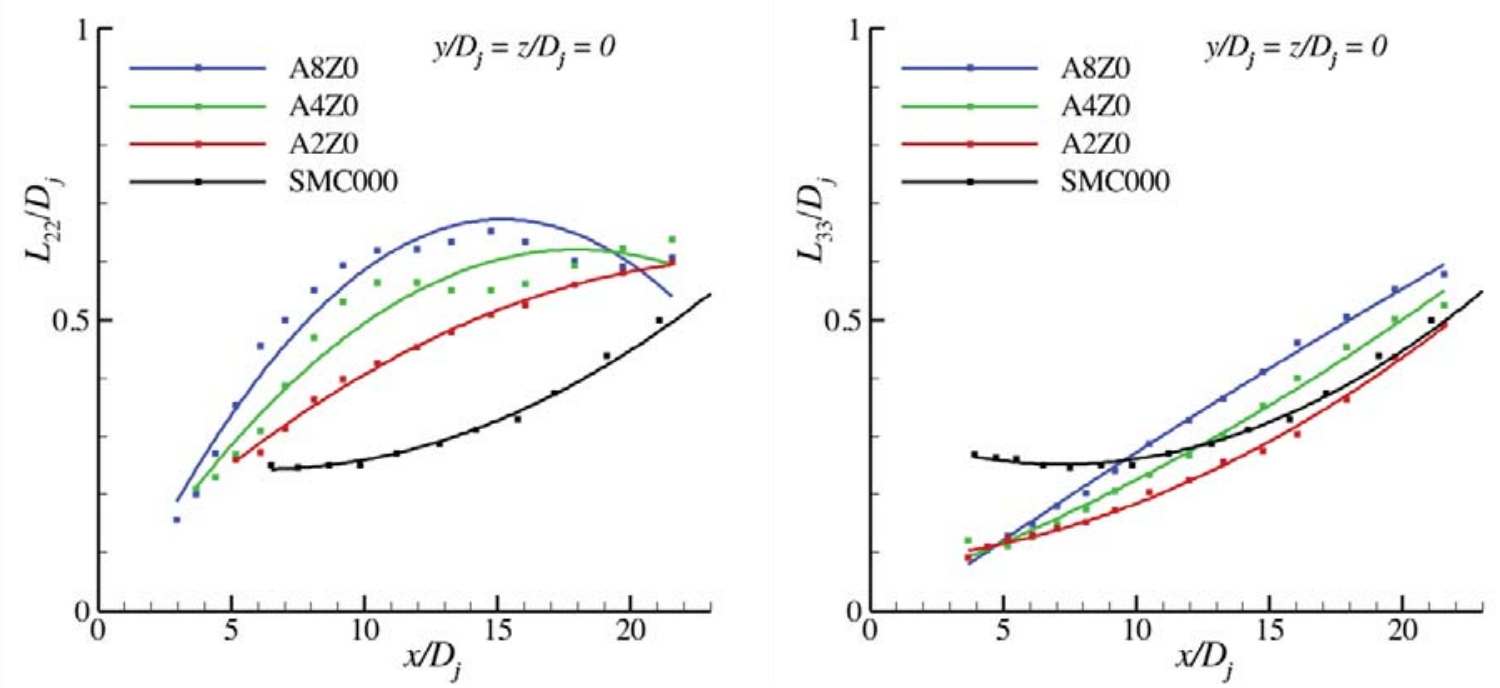

Figure 23 Longitudinal cross-stream lengthscales $L_{22}$ (left) and $L_{33}$ (right) along centerline of rectangular nozzles with $M a=0.9$, unheated flow.

\section{Discussion}

In trying to synthesize the impact of aspect ratio on the flow and turbulence statistics it is tempting to say that increasing the aspect ratio shortens the potential core and that the impact on 
other statistics falls out from this observation. Most of the mean and variance statistics agree with this view. As mentioned above, if the minor axis dimension instead of equivalent diameter normalizes the axial scales then many of the turbulence statistics come together better. For instance, in Figure 24 the mean velocity profiles along the centerlines of the nozzles have the same potential core length when plotted against $x / h$. The variance of velocity along the centerlines also show improvement in matching the rise associated with the end of the potential core, but the rescaling with minor axis height does not collapse the peak locations. Perhaps the development of the plume downstream of the potential core is more dictated by equivalent diameter since the jets have become axisymmetric at this distance downstream.

The ratio of cross-stream to axial turbulent kinetic energy components in Figure 25 do show a better collapsed scaling with nozzle height than with equivalent diameter. The trend toward isotropy of the turbulence components seems to become more universal. On the other hand, the axial longitudinal lengthscales $L_{11}$ shown in Figure 21 could not be made more universal by a rescaling, as shown in Figure 26.

Clearly it is not possible to collapse all the rectangular jet statistics to a single plot by a simple normalization; however, it is possible that many measures, especially those in the potential core region, can be collapsed by rescaling by minor exit height rather than equivalent area.
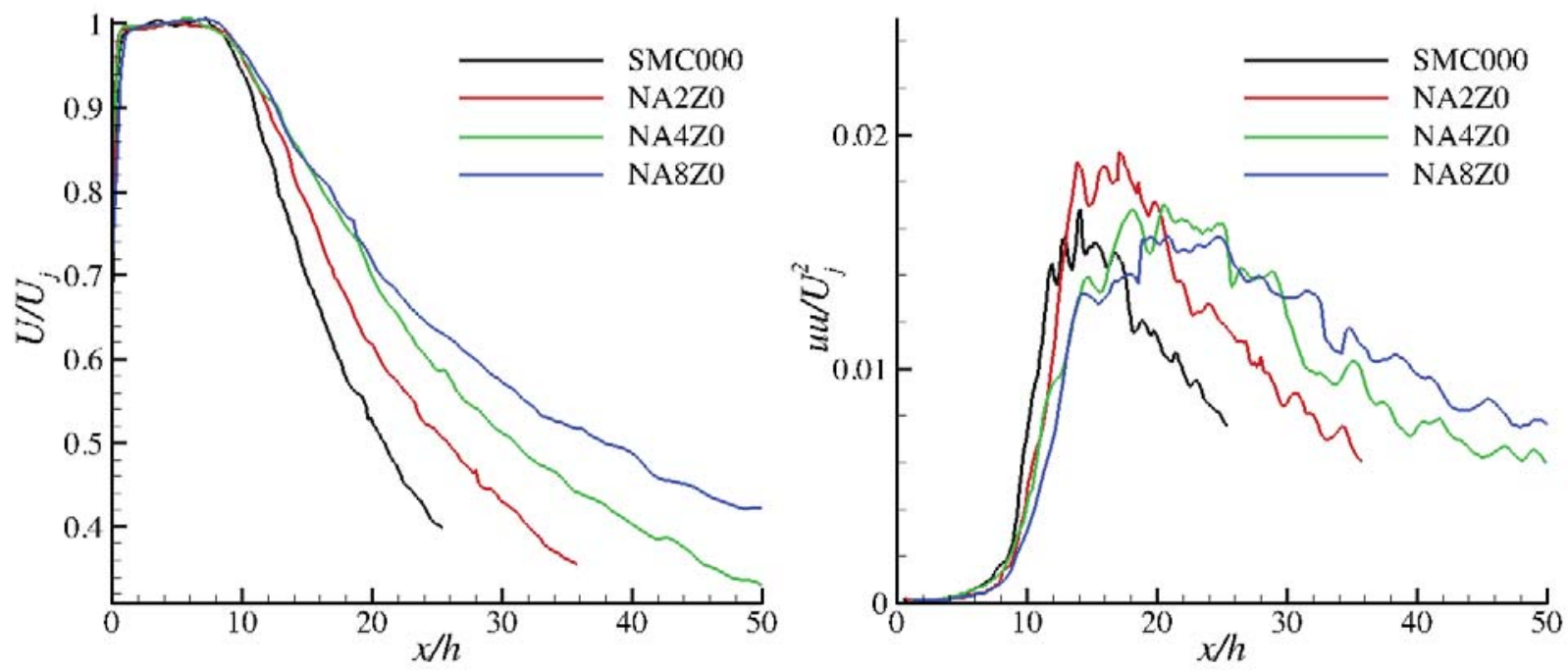

Figure 24 Mean and variance of axial velocity along jet centerlines with axial distance normalized by minor axis height $h$. Note that $h$ is chosen for SMCOOO as if the jet were a 1:1 (square) nozzle. Ma =0.9, unheated flow. 


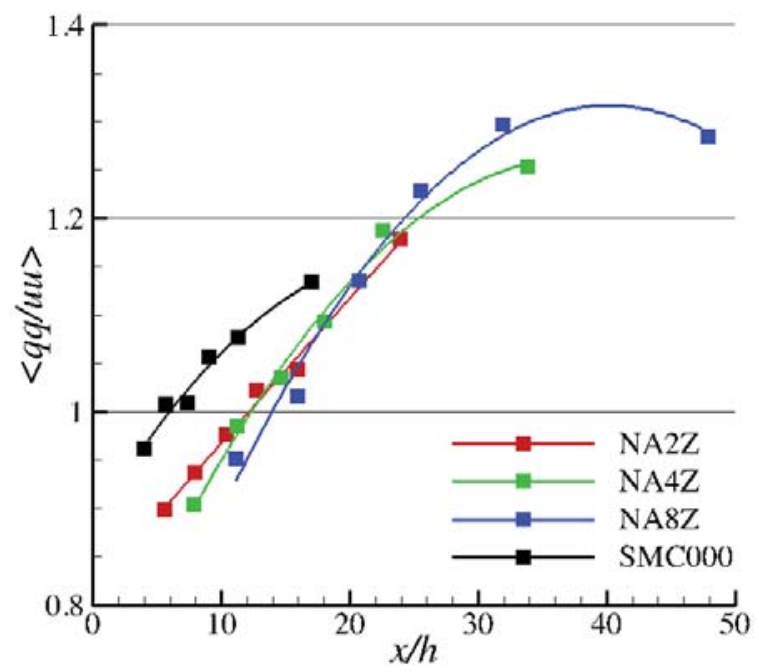

Figure 25 Ratio of cross-stream to axial components of turbulent kinetic energy for beveled nozzles, plotted against axial distance normalized by minor axis height $h . M a=0.9$, unheated flow. Compare with Figure 14.

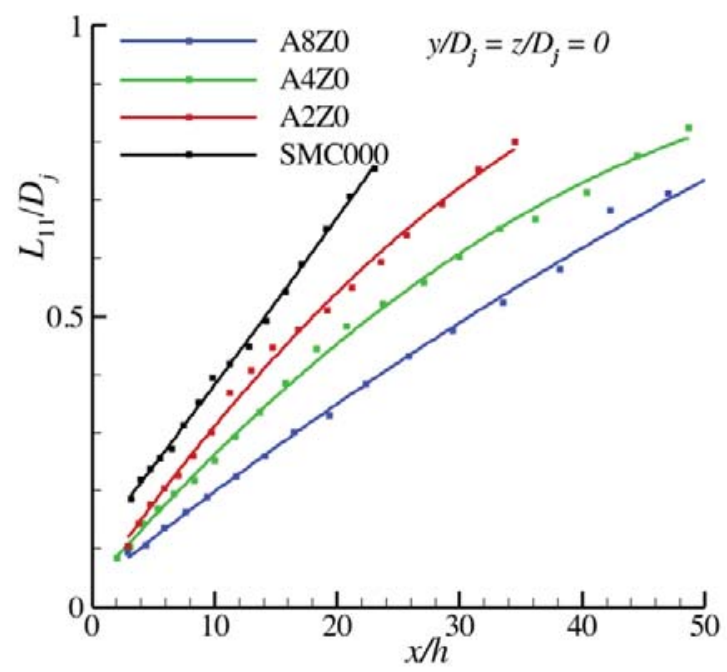

Figure 26 Longitudinal lengthscales $L_{11}, L_{22}, L_{33}$, along centerline three aspect ratio jets and round jet, plotted against axial distance normalized by minor axis height $h . M a=0.9$, unheated flow.

In a previous report ${ }^{5}$, the noise of rectangular nozzles was found to increase with aspect ratio over much of the high frequency range. From the measurements presented above this seems consistent with a shortening of the jet potential core, forcing more turbulent kinetic energy in regions of smaller timescales near the nozzle. There were significant changes in the azimuthal distribution of the noise, perhaps best described as changes in the polar directivity with azimuthal angle. Without extensive invocation of an acoustic analogy theory it is difficult to attribute any of the features of the turbulence measured here with the azimuthal dependence. In 
fact, to really establish the acoustic significance of the anisotropies measured in this paper requires that they be implemented in acoustic analogy models.

\section{Summary}

Rectangular nozzles of aspect ratio 2:1, 4:1, and 8:1 were constructed with care to assure that the exit velocity profile was uniform after the round to rectangular transition and contraction. Extending the wide edge of one side to create beveled nozzles created additional nozzles with the same contraction. The nozzles were tested for far-field acoustics and flow field turbulence, the former showing that increasing the aspect ratio increased noise at high frequencies. Turbulent velocity measurements reported here show that the design goals of uniform exit velocity profile were met, provide data for validation of CFD results, and guide assumptions for modeling of spatial correlations used in acoustic analogy noise prediction codes. Turbulent kinetic energy profiles can be compared with CFD results. All three components of the turbulence, their relationships and distributions have been presented for rectangular nozzles and contrasted with the same measurements in a round jet. Similarly, lengthscales have been computed and compared to show the impact of the nozzle geometry on these statistics. Increasing the aspect ratio decreases the length of the potential core, with a corresponding increase in turbulence with the increased mixing, and an axial foreshortening of other turbulent fields. The main anisotropy noted is in the velocity component aligned with the minor axis. Statistics of this component show an increase in strong activity and increased lengthscales in this direction indicate that large-scale jet flapping is dominant in this direction, potentially causing an azimuthal dependence in noise production.

\section{Acknowledgments}

Supersonics Project of the NASA Fundamental Aeronautics Program supported this work. Cliff Brown and Khairul Zaman provided in-depth critiques of the work.

\section{References}

\footnotetext{
${ }^{1}$ Bridges, J., Khavaran, A., and Hunter, C.G. "Jet Noise," in Assessment of Aircraft Noise Prediction, (ed Milo Dahl) NASA/TP-2012-215653, 2012.

${ }^{2}$ Goldstein, M. E., and Leib, S. J., "The aeroacoustics of slowly diverging supersonics jets", Journal of Fluid Mechanics, 600, pp. 291-337, 2008.

${ }^{3}$ Batchelor, G.K., The Theory of Homogeneous Turbulence, Cambridge University Press, 1953.

${ }^{4}$ Frate, F.C. and Bridges, J., "Extensible Rectangular Nozzle Model System," AIAA Paper 2011-0975, January 2011.
} 
${ }^{5}$ Bridges, J. "Acoustic Measurements of Rectangular Nozzles with Bevel," AIAA Paper 2012-2252, June 2012.

${ }^{6}$ Balsa, T.F., Gliebe, P.R., Kantola, R.A., Mani, R., Stringas, E.J., and Want, F.C.F., "High Velocity Jet Noise Source Location and Reduction: Task 2 - Theoretical Developments And Basic Experiments," FAA-RD-76-79, May 1978.

${ }^{7}$ Massey, K.C., Ahuja, K.K., and Gaeta, R., "Noise Scaling for Unheated Low Aspect Ratio Rectangular Jets," AIAA Paper 2004-2946, May 2004.

${ }^{8}$ Bridges, J. and Wernet, M.P. "The NASA Subsonic Jet Particle Image Velocimetry (PIV) Dataset," NASA/TM-2011-216807, November 2011.

${ }^{9}$ Bridges, J, and Wernet, M.P. "Validating Large-Eddy Simulation for Jet Aeroacoustics," J Propulsion Power 28, (2), pp 226-234, 2012.

${ }^{10}$ M. Harper-Bourne, "Jet noise turbulence measurements," AIAA Paper 2003-3214, 2003.

${ }^{11}$ P.J. Morris, \& K.B.M.Q. Zaman, Velocity measurements in jets with application to noise source modeling, Journal of Sound and Vibration (2009). 